\title{
Fällt die Wahlentscheidung immer später? Die Entwicklung des Zeitpunkts der Wahlentscheidung bei den Bundestagswahlen 1969 bis 2009
}

\author{
Thomas Plischke
}

Do voters decide increasingly late? Changes in the timing of final vote decision in German Federal elections 1969-2009

Abstract: Survey data indicate that German voters make their final vote decision increasingly late. However, doubts persist with regard to the validity of time-of-decision measurement which is based on voters' retrospective self-assessments. In this article, it is examined whether the increase in the share of late decisions can be replicated when applying an alternative measure of time-of-vote decision, thereby making use of panel data of eight German federal elections between 1969 and 2009. It will be demonstrated that the recall measurement lacks validity. Furthermore, the increase in the share of late decisions turns out to be much smaller than previously assumed.

Keywords: Time of vote decision, late deciders, German federal elections, voting behaviour, survey research

Schlagwörter: Zeitpunkt der Wahlentscheidung, Spätentscheider, Bundestagswahlen, Wahlverhalten, Umfrageforschung

\section{Einleitung'}

Man stelle sich eine Demokratie vor, in der bei jeder Wahl bereits Monate vorher feststünde, für welche Parteien sich die Bürger des Landes entscheiden würden. Noch bevor die Parteien überhaupt den Wahlkampf begönnen, stände das Wahlergebnis bereits fest und kein Wahlkampfereignis hätte die Chance, das Ergebnis zu beeinflussen. Natürlich würde sich in einer solchen Demokratie zwangsläufig die Frage stellen, warum Parteien offenkundig belanglose Wahlkämpfe überhaupt führen sollten. Denn wenn sich Wähler selbst durch gute Argumente nicht zu einem Sinneswandel bewegen lassen würden, worin läge dann der Sinn des politischen Wettbewerbs?

1 Der vorliegende Aufsatz ist eine gekürzte Fassung eines Teils meiner Doktorarbeit, die im Januar 2014 mit dem Titel „Wann Wähler entscheiden. Abläufe von Entscheidungsprozessen und der Zeitpunkt der Wahlentscheidung “ im Nomos-Verlag erscheint. Wertvolle Anregungen zu diesem Aufsatz erhielt ich von den Teilnehmern des Forschungskolloquiums des Lehrstuhls für Vergleichende Politische Verhaltensforschung, dem „Young Researcher Network“ der „German Longitudinal Election Study“ und den Teilnehmern der Tagung des „AK Wahlen und politische Einstellungen“ in Frankfurt 2012. Gedankt sei auch Maria Müller, Frederike Oschinsky und Louisa Plasberg für hervorragende Forschungsassistenz. 


\section{Abbildung 1: Die Entwicklung des Zeitpunkts der Wahlentscheidung bei Bundes- tagswahlen, 1965-2009}

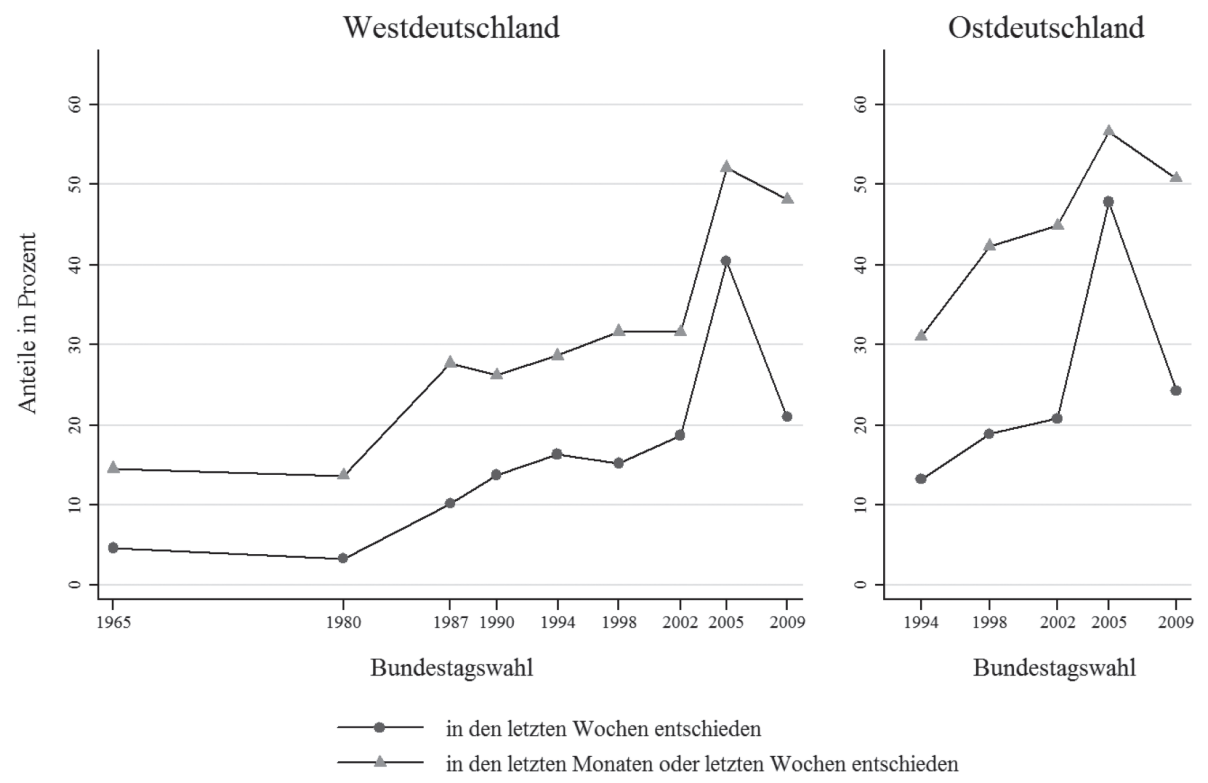

Daten: Deutsches Institut für Volksumfragen 1965; Fuchs et al. 1980; Berger et al. 1987b; Forschungsgruppe Wahlen 1990; Berger et al. 1994a; Berger et al. 1994b; Falter et al. 1998; Falter et al. 2002; Weßels 2005; Rattinger et al. 2009a.

Man könnte meinen, eine solche Demokratie wäre nur Fiktion. Wenn man jedoch den Daten deutscher Wahlstudien aus den Sechziger- und Siebzigerjahren Glauben schenkt, dann waren die damaligen Bundestagswahlen nicht weit von dieser Fiktion entfernt. Damals trafen nach eigenen Angaben nur etwa 5 Prozent der Wähler ihre Entscheidung innerhalb der letzten Wochen vor der Wahl. Hingegen äußerten bis zu 85 Prozent der Wähler, dass sie ihre Wahlentscheidung bereits „seit langem“ getroffen hätten (vgl. Abbildung 1). Der Akt des politischen Wählens, so der Kommentar von Dalton und Bürklin (2003, S. 66), konnte damals besser mit dem Begriff „Gewohnheit" charakterisiert werden als durch das Wort „Wahl-Entscheidung“.

Jüngere Wahlstudien zeichnen ein deutlich verändertes Bild. In Deutschland, wie in anderen westlichen Demokratien auch (Dalton et al. 2000; McAllister 2002; Plasser et al. 2003; Irwin u. Van Holsteyn 2008), besteht ein Trend zu späteren Wahlentscheidungen. Bei den Bundestagswahlen 2005 und 2009 bekundeten mehr als 50 Prozent der Wähler in Ost- und Westdeutschland, sie hätten ihre Wahlentscheidung in den letzten Monaten oder Wochen vor der Wahl getroffen. Die Implikation, die sich aus dieser Entwicklung für das Wirkungspotenzial von Wahlkämpfen oder der Medienberichterstattung kurz vor der Wahl ergibt, lässt sich anhand des obigen Gedankenexperiments leicht verdeutlichen: Das Potenzial für Wahlkampfeffekte ist enorm gestiegen (Chaffee u. Rimal 1996; Schmitt-Beck 2003). 
Doch entspricht die festgestellte Zunahme von späten Wahlentscheidungen der Realität? Die in Abbildung 1 dargestellten Messwerte beruhen auf Antworten auf eine Rückerinnerungsfrage, die den folgenden Wortlaut hat:

„Wann haben Sie sich entschieden, wie Sie bei dieser Bundestagswahl gewählt haben? Stand das schon seit langem fest, stand das seit ein paar Monaten fest, haben Sie sich in den letzten Wochen entschieden, den letzten Tagen vor der Wahl entschieden oder erst am Wahltag selbst? “ (Frageformulierung aus der Querschnittsstudie der „German Longitudinal Election Study“; Rattinger et al. 2009a)

Allein in der Wahlforschung stößt man auf eine fast unüberschaubare Literatur, in der die Validität von Rückerinnerungsfragen grundsätzlich angezweifelt wird (Himmelweit et al. 1978, Berger et al. 1983a, S. 52; van der Eijk u. Niemöller 1983, S. 140; Juhász 1993; Waldahl u. Aardal 2000; Schoen 2003, S. 140-151). Auch in Bezug auf die obige Rückerinnerungsfrage ist zu vermuten, dass eine valide Antwort ein hohes Ausmaß an Erinnerungsvermögen und Meta-Kognition seitens der Befragten erfordert. In der US-amerikanischen Wahlforschung wurde bereits empirisch gezeigt, dass Antworten auf die Zeitpunkt-Frage stark fehlerbehaftet und sogar systematisch verzerrt sind (Plumb 1986; Chaffee u. Rimal 1996; Kogen u. Gottfried 2011); anders jedoch verhält es sich in Kanada (Fournier et al. 2001, 2004). In Deutschland kommen erste Untersuchungen zu gegensätzlichen Einschätzungen: Schmitt-Beck und Partheymüller (2012, S. 314) sehen die Validität der Rückerinnerungsfrage als gegeben an, während Reinemann et al. (2013, S. 119-120) erhebliche Messfehler feststellen.

Glücklicherweise ist die Forschung nicht ausschließlich auf die retrospektive Selbsteinstufung der Befragten angewiesen. Es ist möglich, den Entscheidungszeitpunkt von Wählern näherungsweise mit einer alternativen Methode zu bestimmen, die im Folgenden als „Panelmethode“ bezeichnet wird. Im vorliegenden Aufsatz wird diese Methode angewandt, um die beiden folgenden Fragen zu untersuchen: Erstens, lässt sich die Zunahme später Wahlentscheidungen auch mit Paneldaten belegen? Zweitens, ist die Rückerinnerungsmethode ein valides Maß für die Messung des Entscheidungszeitpunkts? Für die Bearbeitung dieser Fragestellungen stehen acht Wahlkampf-Panelstudien aus einem Zeitraum von 1969 bis 2009 zur Verfügung. Die Ergebnisse stellen die Validität der Rückerinnerungsmethode sowie die Existenz eines zeitlichen Trends in Frage.

\section{Die Panelmethode}

Durch ihre erstmalige Anwendung in der berühmten „Erie-County-Studie“ (Lazarsfeld et al. 1944) ist die Panelmethode das ältere der beiden Verfahren zur Messung des Entscheidungszeitpunkts. Die Forschergruppe führte damals eine sieben Wellen umfassende Wiederholungsbefragung durch, wobei Personen aus dem Erie County in Ohio bis zu sechs Mal vor der Wahl und einmal nach der Wahl befragt wurden. Als time of final decision wurde dabei jener Befragungszeitpunkt definiert, ab dem sich eine einmal geäußerte Wahlintention bis hin zu ihrer Realisierung am Wahltag nicht mehr veränderte. Auf dieser Grundlage wurden die Wähler dann in drei Kategorien eingeteilt: Die Mai-Wähler, deren Entschei- 
dung bereits in der ersten Panelwelle feststand; die Juni-bis-August-Wähler, deren Entscheidung sich in den Panelwellen 2 bis 4 herausbildete; und die Septemberbis-November-Wähler, die sich erst in den letzten Panelwellen endgültig auf eine Partei festlegten.

Tabelle 1: Die Logik der Panelmethode, verdeutlicht anhand drei exemplarischer Verlaufsmuster von Wahlintentionen in einem siebenwelligen Panel mit zwei Parteien

\begin{tabular}{|c|ccccccc|}
\hline id & $\begin{array}{c}\text { Welle 1 } \\
\text { (Vorwahl) }\end{array}$ & $\begin{array}{c}\text { Welle 2 } \\
(\text { Vorwahl })\end{array}$ & $\begin{array}{c}\text { Welle 3 } \\
\text { (Vorwahl) }\end{array}$ & $\begin{array}{c}\text { Welle 4 } \\
\text { (Vorwahl) }\end{array}$ & $\begin{array}{c}\text { Welle 5 } \\
\text { (Vorwahl) }\end{array}$ & $\begin{array}{c}\text { Welle 6 } \\
\text { (Vorwahl) }\end{array}$ & $\begin{array}{c}\text { Welle 7 } \\
\text { (Nachwahl) }\end{array}$ \\
\hline 1 & weiß nicht & weiß nicht & weiß nicht & Partei A & Partei A & Partei A & Partei A \\
2 & Partei A & Partei A & Partei B & Partei B & Partei B & Partei B & Partei B \\
3 & Nichtwahl & Nichtwahl & Nichtwahl Nichtwahl & Partei B & Partei B & Partei B \\
\hline
\end{tabular}

Fettdruck: Gemessener Zeitpunkt der Wahlentscheidung gemäß der Panelmethode

Tabelle 1 verdeutlicht diese Logik anhand drei fiktiver Befragten. Der Befragte in Zeile 1 war in den ersten drei Wellen unentschlossen, wies jedoch in der vierten Welle erstmals eine Wahlintention zugunsten Partei A auf, die er später dann auch wählte. Nach der Panelmethode gilt demnach die vierte Befragung als Entscheidungszeitpunkt. Dieselbe Logik lässt sich auf die Befragten 2 und 3 anwenden, obwohl diese auf anderen Wegen zu ihren endgültigen Wahlentscheidungen gelangten: Der Befragte 2 änderte seine ursprüngliche Wahlintention in Welle 3, der Befragte 3 entschloss sich erst in Welle 5, überhaupt an der Wahl teilzunehmen. Somit sind in Tabelle 1 die drei empirischen Korrelate „später"2 Wahlentscheidungen beschrieben: Wähler können sich a) spät entscheiden, an der Wahl teilzunehmen, b) spät ihre ursprüngliche Wahlintention revidieren oder sie können c) lange unentschlossen sein. Wie im Folgenden gezeigt wird, ist es ein wesentlicher Vorteil der Panelmethode gegenüber der Rückerinnerungsmethode, dass sich diese drei unmittelbaren Manifestationen später Wahlentscheidungen voneinander abgrenzen lassen.

Anhand der Panelmethode lässt sich der Entscheidungszeitpunkt nicht exakt, sondern allenfalls näherungsweise bestimmen. Die Wahlentscheidung des ersten Befragten fiel vermutlich nicht erst am Tag des vierten Interviews, sondern irgendwann im Zeitraum zwischen der dritten und der vierten Befragung. Darüber hinaus basiert die Panelmethode auf der Prämisse, dass zwei identische Wahlintentionen, die in aufeinander folgenden Panelwellen geäußert wurden, tatsächlich auf Stabilität dieser Wahlintentionen zwischen den beiden Erhebungen hindeuten. Dementgegen wäre es beispielsweise vorstellbar, dass sich Befragter 2 zwischen der vierten und der fünften Welle umentschied und für eine kurze Zeit für Partei A stimmen wollte, diese Entscheidung aber kurz darauf wieder revidierte und zu

2 Die Begriffe „früh“ und „spät“ werden im Folgenden nicht weiter definiert. Sie dienen sprachlich lediglich dazu, Tendenzen und Relationen auszudrücken. 
seiner ursprünglichen Intention zurückkehrte. Hätte man diesen Prozess beobachtet, wäre das Datum der fünften Welle der Zeitpunkt der Wahlentscheidung und nicht das Datum der dritten Welle. Da sich nie ausschließen lässt, dass zwischen den Panelwellen unbeobachtete Veränderungen stattfanden, lässt sich der Zeitpunkt der Wahlentscheidung über die Panelmethode strikt genommen niemals positiv bestimmen, da es prinzipiell immer möglich ist, dass der tatsächliche Entscheidungszeitpunkt später stattfand.

Der wesentliche Vorteil der Panelmethode besteht in ihrer Eigenschaft, eine negative Identifikation des Entscheidungszeitpunkts zu ermöglichen. Darunter ist die Identifikation jener Zeitpunkte zu verstehen, an denen die endgültige Entscheidung einer Person objektiv noch nicht endgültig gefallen war. Auch dieser Punkt lässt sich beispielhaft anhand von Tabelle 1 verdeutlichen: Weil die Person in Zeile 1 in der ersten Welle auf die Frage nach der Wahlintention mit „weiß nicht" antwortete, kann mit Sicherheit gesagt werden, dass sie zu diesem Zeitpunkt noch nicht endgültig entschlossen war. Ebenso war Befragter 2 in der zweiten Welle noch nicht endgültig entschlossen, denn er äußerte eine Wahlabsicht für eine Partei, die er später nicht wählte. Die auf diese Weise ermittelten Zeitpunkte der „Nicht-Entscheidung“ können dazu verwendet werden, um die Antworten auf die Rückerinnerungsmethode zu validieren. Wenn sich etwa herausstellen würde, dass zahlreiche Befragte einer Panelstudie einige Tage vor der Wahl noch keine Wahlintention für die später gewählte Partei aufwiesen, dieselben Personen jedoch nach der Wahl bekundeten, bereits „seit langer Zeit“ entschieden gewesen zu sein, so erwüchsen berechtigte Zweifel an der Validität der Rückerinnerungsfrage. Die in Abschnitt 5 durchgeführte Analyse basiert auf diesem Prinzip.

\section{Daten und Operationalisierung}

Insgesamt liegen acht Datensätze vor, anhand derer sich der Zeitpunkt der Wahlentscheidung über die Panelmethode operationalisieren lässt (Klingemann u. Pappi 1969; Berger et al. 1972; Berger et al. 1976; Berger et al. 1983b; Berger et al. 1987a; Kaase et al. 1990; Schmitt-Beck u. Faas 2005; Rattinger et al. 2009b). Diese Datensätze decken insgesamt einen Zeitraum von 40 Jahren ab, wobei einige zeitliche Diskontinuitäten bestehen: Für die Bundestagswahlen 1980, 1994, 1998 und 2002 liegen keine Panelstudien vor, in denen der Zeitpunkt der Wahlentscheidung in der Nachwahl-Panelwelle erhoben wurde.

Die Studien weisen Unterschiede im Design auf. Erstens unterscheidet sich die Anzahl der Vorwahlbefragungen: Die Panelstudien 1965, 2005 und 2009 weisen nur eine einzige Vorwahlbefragung auf, während die Teilnehmer an der Panelstudie 1990 bis zu dreimal vor der Wahl interviewt wurden. Alle anderen Studien verfügen über zwei Vorwahlbefragungen. Zweitens variiert die zeitliche Taktung der Befragungen erheblich: Während die Vorwahlinterviews zur Wahl 1969 in den letzten drei Wochen vor der Wahl durchgeführt wurden, erstreckten sich die drei Vorwahlbefragungen 1990 über das gesamte Jahr. Drittens bestehen Unterschiede im Erhebungsmodus: Alle Interviews bis einschließlich 1990 wurden face-to-face durchgeführt, die Erhebungen 2005 und 2009 hingegen telefonisch. Viertens handelt es sich bei den Studien aus den Jahren 2005 und 2009 um ein sogenanntes 
Rolling-Cross-Section-Design (RCS-Design [Schmitt-Beck et al. 2006]). Das Design einer RCS-Studie ist so angelegt, dass an jedem Tag eines festgelegten Zeitraumes eine zufällig ausgewählte Stichprobe von Befragten interviewt wird. Aus dem Vergleich aller täglichen Querschnitte resultiert eine sehr dichte Zeitreihe von Beobachtungen, mit der die Effekte von tagesaktuellen Ereignissen auf aggregierte Wählermerkmale untersucht werden können. Da die tagesaktuellen Stichproben sehr klein (etwa 90 Personen pro Tag) und somit anfällig für zufallsbedingte Fehler sind, werden die Umfragedaten aus jeder einzelnen Woche zusammengefasst.

Vor allem die unterschiedlichen Befragungsfrequenzen in den einzelnen Panelstudien stellen ein Problem für die Vergleichbarkeit der Ergebnisse dar. Wenn man eine Person dreimal vor einer Wahl interviewen würde, läge die Wahrscheinlichkeit, dass sie in diesem Zeitraum mindestens einmal nicht wählen wollte, unentschlossen wäre oder die präferierte Partei wechseln würde, wesentlich höher, als wenn man die Person im selben Zeitraum nur ein einziges Mal interviewen würde. Um die Vergleichbarkeit der Ergebnisse zwischen den Studien zu gewährleisten, wurden bei der Berechnung der Anteilswerte für einen bestimmten Befragungszeitraum alle Daten aus nachfolgenden Vorwahlwellen ignoriert. Wenn beispielsweise eine Person in der ersten Vorwahlbefragung eine mit ihrer späteren Parteiwahl konsistente Wahlabsicht nannte, wurde sie selbst dann als „entschlossen" klassifiziert, wenn aus nachfolgenden Befragungen ersichtlich wurde, dass sie ihre Wahlabsicht noch einmal veränderte. Das führt zwar dazu, dass der Anteil noch nicht endgültig entschlossener Wähler unterschätzt wird. Zumindest wird dadurch aber Vergleichbarkeit hergestellt, weil die Tendenz der Unterschätzung in allen verwendeten Panelstudien in einem gleichen Ausmaß besteht.

Um die Anteilswerte der noch nicht endgültig entschlossenen Wähler zu bestimmen, wurden für alle Vorwahlbefragungen die Anteile der tatsächlichen Wähler ermittelt, die zum Zeitpunkt der Vorwahlbefragung a) noch nicht an der Wahl teilnehmen wollten, b) als Wahlintention eine Partei nannten, die nicht mit der später gewählten Partei übereinstimmte oder c) noch unentschlossen waren. Da in Panelstudien systematische Panelausfälle auftreten, wurden fehlende Werte anhand von propensity score matching (Bacher 2002) ersetzt. Dazu wurde die sogenannte „Zwillingsmethode“ angewendet: Es wurden Paare an Befragten identifiziert, die möglichst in allen für den Befragungszeitpunkt relevanten Merkmalen identisch sind, sich jedoch nur in der Teilnahme an einer bestimmten Welle voneinander unterscheiden. Dann wurde der fehlende Wert durch den vorhandenen Wert des „Zwillings“ ersetzt. Durch die Ersetzung erhöhten sich die Anteile der noch nicht entschlossenen Wähler leicht, was aufgrund theoretischer Überlegungen zu erwarten war. 


\section{Die Entwicklung des Zeitpunkts der Wahlentscheidung nach der Panelmethode}

Lässt sich eine Zunahme von späten Wahlentscheidungen beobachten, wenn man zur Messung des Entscheidungszeitpunkts statt der Rückerinnerungsmethode die Panelmethode verwendet? Es sei daran erinnert, dass die Messmethoden unterschiedlich funktionieren: Während die Rückerinnerungsmethode den Anteil der zu einem gegebenen Zeitraum endgültig getroffenen Entscheidungen quantifiziert, gibt die Panelmethode Auskunft über den Anteil der zu einem bestimmten Zeitpunkt noch nicht endgültigen Entscheidungen. Wenn beide Methoden valide sind, sollte sich die Zunahme später Entscheidungen sowohl bei der Rückerinnerungsfrage als auch in den Paneldaten manifestieren: Es wäre zu erwarten, dass der für einen gegebenen Zeitraum vor der Wahl gemessene Anteil der noch nicht entschlossenen Wähler über die Jahrzehnte hinweg stark zunimmt.

In Abbildung 2 sind die Anteilswerte der noch nicht entschlossenen Wähler in Abhängigkeit von der Nähe zum Wahltag abgetragen. Die Tatsache, dass die Anteilswerte nicht als Punkte, sondern als horizontale Linien dargestellt werden, trägt dem Umstand Rechnung, dass sich die Datenerhebung bei einzelnen Befragungswellen über mehrere Wochen erstreckte. Es ist daher nur möglich, Anteilswerte für den gesamten Erhebungszeitraum anzugeben. Eine Ausnahme sind die RCS-Studien 2005 und 2009, in denen in jeder Woche ein repräsentativer Querschnitt der Gesamtbevölkerung interviewt wurde, sodass für diese Jahre die Anteilswerte wochenweise variieren.

Im Zeitraum von 1969 bis 1990 variierte der Anteil der noch nicht entschlossenen Wähler überraschenderweise nur wenig. 16 bis 30 Wochen vor der Wahl waren etwa 30 bis 35 Prozent der Wähler objektiv noch nicht entschlossen, für die letzten acht Wochen vor der Wahl reduzierte sich der Anteil auf 22 bis 29 Prozent. Ein Ausreißer ist die Wahl 1987: Hier lag der Anteil der noch nicht entschlossenen Wähler wenige Wochen vor der Wahl bei nur 17 Prozent. Insgesamt lässt sich vor 1990 jedoch kein zeitlicher Trend spät getroffener Entscheidungen nachweisen. Dies steht im Widerspruch zu den Befunden in Abbildung 1.

Ebenfalls in Widerspruch zu Abbildung 1 stehen die absoluten Anteilswerte. Es sei daran erinnert, dass 1965 und 1980 jeweils maximal nur 5 Prozent der Wähler angaben, sie hätten ihre Wahlentscheidung innerhalb der letzten Wochen vor der Wahl getroffen. Dieser Anteil lässt sich mit Paneldaten aus diesem Zeitraum nicht belegen: Im Jahr 1969 wiesen zwei bis drei Wochen vor der Wahl mindestens 28 Prozent der Wähler noch keine Wahlintention auf, die sich mit ihrer späteren Parteiwahl deckte. Ähnliche Anteilswerte sind auch für die Wahlstudien 1972, 1976 und 1983 zu verzeichnen. Die tatsächlichen Anteile liegen sogar noch höher, da die Panelmethode den Anteil der noch nicht endgültig getroffenen Entscheidungen unterschätzt. 


\section{Abbildung 2: Anteilswerte der noch nicht entschlossenen Wähler in Abhängig- keit vom zeitlichen Abstand zur Wahl}

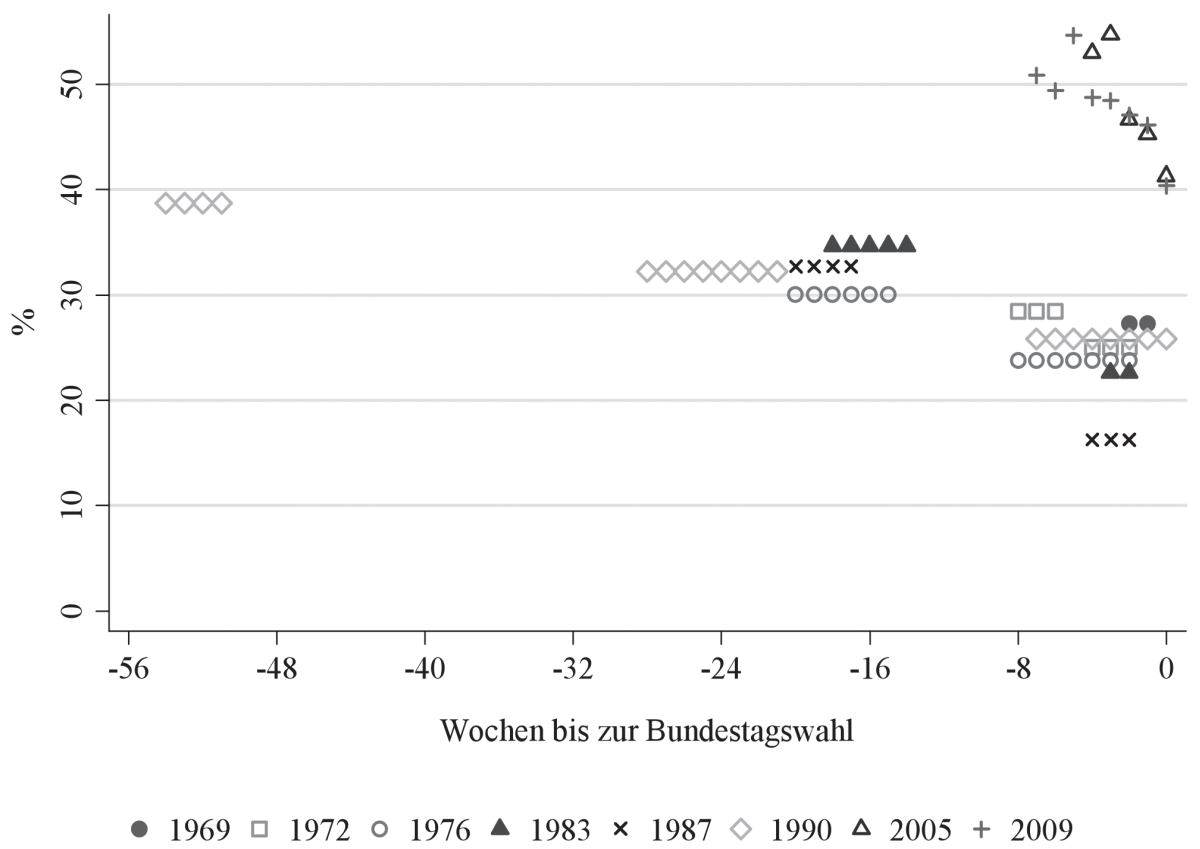

Daten: Klingemann u. Pappi 1969; Berger et al. 1972; Berger et al. 1976; Berger et al. 1983b; Berger et al. 1987a; Kaase et al. 1990; Schmitt-Beck u. Faas 2005; Rattinger et al. 2009b.

Hingegen lässt sich die Existenz eines Anstiegs später Wahlentscheidungen zwischen 1990 und 2005 mit den Paneldaten belegen. Bei den Bundestagswahlen 2005 und 2009 lag der Anteil der noch nicht endgültig entschlossenen Wähler eine bis acht Wochen vor der Wahl um etwa 10 bis 20 Prozentpunkte höher als in allen anderen Wahlstudien. ${ }^{3}$ Das entspricht etwa dem anhand der Rückerinnerungsmethode festgestellten Anstieg der in den „letzten Wochen“ getroffenen Entscheidungen bei der Bundestagswahl 2005. Während allerdings in Abbildung 1 für 2009 wieder ein deutlicher Rückgang der in den „letzten Wochen“ getroffenen Entscheidungen zu beobachten war, ist ein vergleichbarer Rückgang in den Paneldaten nicht zu konstatieren. Vertraut man den Ergebnissen der Panelmethode, so fielen die Entscheidungen 2009 deutlich später, als es die Ergebnisse der Rückerinnerungsmethode suggerieren.

Mit der Panelmethode ist es nun möglich, noch einen Schritt weiterzugehen und zu untersuchen, wodurch die Zunahme später Wahlentscheidungen ausgelöst wurde. Drei unmittelbare Ursachen oder eine Kombination dieser drei Ursachen

3 In den Jahren 2005 und 2009 gab es zwischen Ost- und Westdeutschland keine signifikanten Unterschiede in den Anteilswerten der noch nicht endgültig entschlossenen Wähler. Aus diesem Grund wurde auf eine getrennte Darstellung der ost- und westdeutschen Ergebnisse verzichtet. 
können für die Zunahme verantwortlich sein: Erstens könnte der Anteil der erst kurzfristig mobilisierten Wähler angestiegen sein. Darunter fallen jene Wähler, die im Vorwahlinterview angaben, nicht wählen zu wollen, sich aber dann doch zur Teilnahme entschlossen. Zweitens könnte der Anteil jener Wähler, die im Verlauf des Wahlkampfes ihre Wahlintention von einer Partei zu einer anderen Partei veränderten, zugenommen haben. Drittens könnte die Zunahme später Wahlentscheidungen in einer Zunahme von unentschlossenen Wählern begründet liegen; jene Wähler also, die vor der Wahl auf der Frage nach der Wahlintention mit „weiß nicht“ antworteten. Die Aufschlüsselung der noch nicht entschlossenen Wähler in diese drei Teilgruppen erfolgt in den Abbildung 3a-c.

Wie Abbildung 3a zeigt, kann die Zunahme später Wahlentscheidungen nicht dadurch erklärt werden, dass sich Wähler zunehmend später mobilisieren lassen. Die nicht-mobilisierten Wähler stellen generell nur einen sehr kleinen Teil der späten Entscheider. Selbst ein Jahr vor der Wahl lag ihr Anteil 1990 bei nur 8 Prozent. Mit zunehmender Nähe zum Wahltag besteht eine leichte Tendenz zur Mobilisierung. Bei der Betrachtung der letzten acht Wochen vor der Wahl fällt auf, dass sich ein schwacher zeitlicher Trend identifizieren lässt: Die niedrigsten Anteilswerte stammen aus den Siebzigerjahren, als nahezu alle wahlberechtigten Bürger an der Wahl teilnahmen. Die höchsten Anteilswerte der noch nicht mobilisierten Wähler kommen aus dem Jahr 2009, dem Jahr der niedrigsten Wahlbeteiligung. Doch selbst 2009 lagen die Anteilswerte kurz vor der Wahl noch unterhalb von 10 Prozent.

Auch Präferenzwechsel scheiden als direkte Ursache für den Anstieg später Wahlentscheidungen aus. Zwar geht aus Abbildung $3 \mathrm{~b}$ klar hervor, dass Parteipräferenzwechsel eine wichtige Ursache später Wahlentscheidungen sind, da 10 bis 22 Prozent der Wähler in den letzten Wochen vor der Wahl noch ihre Parteipräferenz wechselten. Jedoch gibt es keine Evidenz dafür, dass der Anteil der in der Vorwahlzeit stattfindenden Präferenzwechsel über die letzten Jahrzehnte hinweg systematisch zunahm. Wenn man den Zeitraum der letzten acht Wochen vor der Wahl betrachtet, so liegt der höchste Anteil der Präferenzwechsel bei 22 Prozent im Jahr 1990. Der geringste Anteil, mit etwas weniger als 10 Prozent, kann für das Jahr 1976 festgestellt werden. Die Bundestagswahl 2005 weist mit 10 bis 15 Prozent vergleichsweise niedrigere Wechselraten auf. Bei der Bundestagswahl 2009 lagen die Wechselraten mit 12 bis 17 Prozent leicht höher.

Es bleibt somit logisch nur noch eine direkte Ursache für die Zunahme später Wahlentscheidungen: Der Anteil der unentschlossenen Wähler nahm zwischen 1990 und 2005 stark zu (Abbildung 3c). Er lag in den frühen Wahlstudien wenige Wochen vor der Wahl bei lediglich 10 Prozent, 1983, 1987 und 1990 sogar bei nur 5 Prozent oder noch weniger. In der Wahlstudie 2005 lag der Anteil unentschlossener Wähler im gleichen Zeitraum bei fast 40 Prozent, 2009 immerhin zwischen 22 und 30 Prozent. 
Abbildung 3: Anteilswerte der Nichtwähler, Parteiwechsel und unentschlossenen Wähler in Abhängigkeit vom zeitlichen Abstand zur Wahl

(a) noch nicht mobilisiert

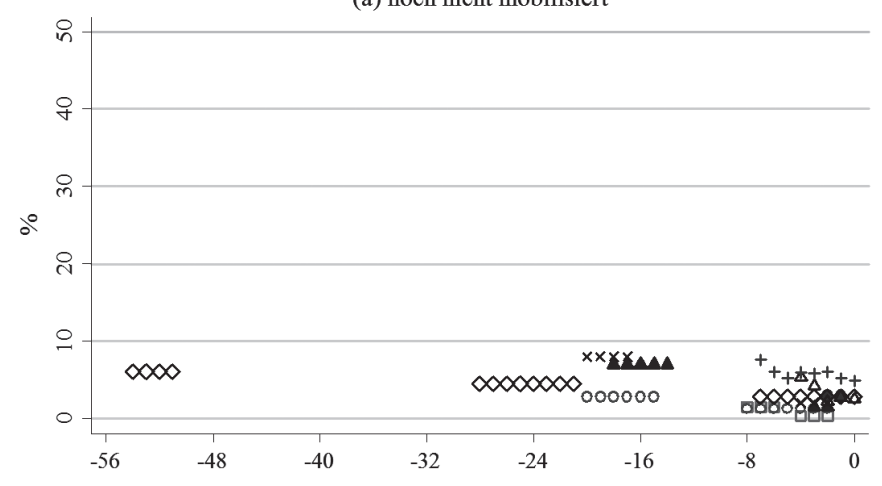

(b) Wahlintention stimmt noch nicht mit Parteiwahl überein

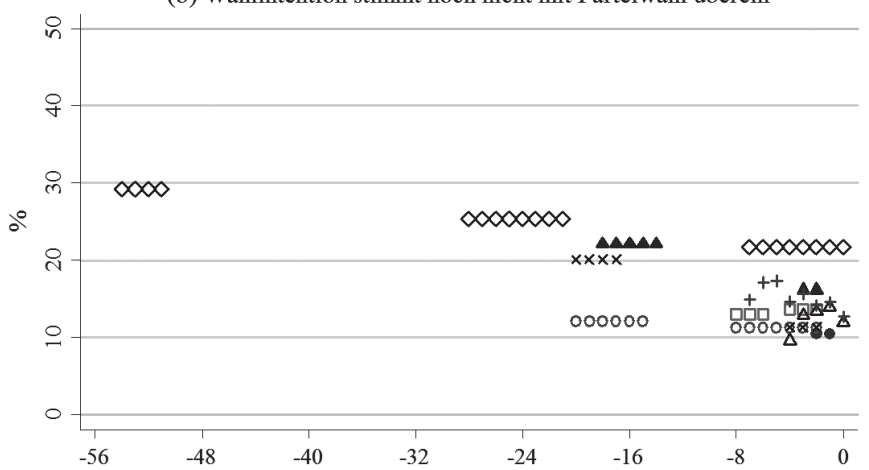

(c) noch unentschlossen

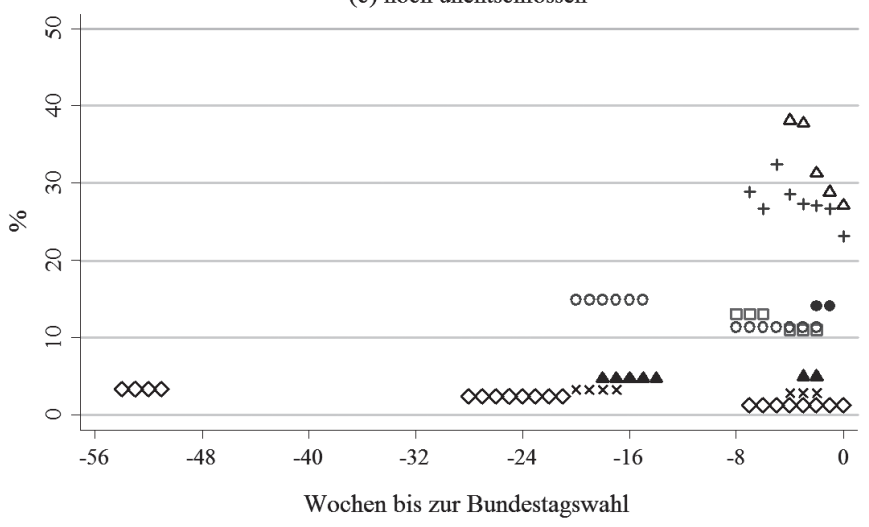

- 1969 口 $1972 \circ 1976$ \& $1983 \times 1987 \diamond 1990 \Delta 2005+2009$

Daten: vgl. Abbildung 2. 
Wie lässt sich die extreme zeitliche Variation in den Anteilen der unentschlossenen Wähler erklären? Die Zunahme unentschlossener Wähler wurde in der Literatur bislang häufig mit dealignment in Verbindung gebracht (z. B. Dalton u. Bürklin 2003; Dalton 2012): Habe früher ein Großteil der Wähler „feststehende Wahlentscheidungen" aufgewiesen, von denen sie nur in Ausnahmefällen abgewichen seien, existiere heutzutage ein stetig wachsendes unabhängiges Wählersegment, das zu Beginn des Wahlkampfes noch keine Parteipräferenz aufweise und sich erst im Verlauf des Wahlkampfes auf eine Partei festlege. Doch dealignment kann nicht den Sprung der Unentschlossenen-Anteile zwischen 1990 und 2005 von etwa 1 bis 2 Prozent auf fast 40 Prozent erklären, da es sich um einen eher graduellen, sich langfristig und langsam ablaufenden Prozess handelt.

$\mathrm{Da}$ inhaltliche Erklärungsansätze nicht überzeugen, ist es naheliegend, die Variation der Unentschlossenen-Anteile auf methodische Unterschiede in den vorliegenden Studien zurückzuführen. In der Tat existieren charakteristische Unterschiede in der Abfrage der Wahlabsicht zwischen den Wahlstudien 1972 bis 1990 auf der einen Seite und den RCS-Studien 2005 und 2009 auf der anderen Seite. Die früheren Panelstudien wurden allesamt face-to-face durchgeführt. Bei der Abfrage der Wahlabsicht hatte dieser Modus den Vorteil, dass den Befragten eine Liste oder ein Stimmzettel vorgelegt werden konnte, auf denen alle Antwortoptionen standen. Die Aufgabe der Befragten bestand lediglich darin, eine Partei der Liste auszuwählen. Man spricht hier von einer gestützten Abfrage der Wahlabsicht. Die RCS-Studien 2005 und 2009 wurden hingegen telefonisch (CATI computer-assisted telephone interviewing) durchgeführt. Da das Vorlegen einer Liste oder eines Stimmzettels bei einer telefonischen Befragung nicht möglich ist, wurde die Wahlabsichtsfrage in den RCS-Studien ungestützt durch eine offene Frage erhoben:

„Bei der Bundestagswahl am 27. September können Sie ja zwei Stimmen vergeben. Die Erststimme für den Kandidaten einer Partei in Ihrem Wahlkreis, die Zweitstimme für eine Partei. Den Wahlkreiskandidaten welcher Partei werden Sie mit Ihrer Erststimme wählen? “ (Antwort des Befragten) „Und welche Partei werden Sie bei der Bundestagswahl mit Ihrer Zweitstimme wählen?” (Frageformulierung aus der RCSStudie 2009: Rattinger et al. 2009b)

Eine offene Frage zur Messung der Wahlintention erhöht den kognitiven Aufwand für die Befragten erheblich. Um zwischen einzelnen Parteien überhaupt abwägen zu können, müssen sich die Befragten die Parteien selbst ins Gedächtnis rufen. Da das Arbeitsgedächtnis von Menschen über eine nur sehr begrenzte Speicherkapazität verfügt (Miller 1956), ist eine Menge „Kopfrechnen“ (Verschieben von Informationen zwischen Arbeits- und Langzeitgedächtnis) erforderlich, um Parteinamen aus dem Langzeitgedächtnis abzurufen, sie zu bewerten und zu vergleichen, um auf diese Weise zu einer Wahlintention zu gelangen. Besonders schwer dürfte diese Aufgabe für Befragte mit nur geringem politischen Wissen sein, da diese seltener über starke Einstellungen verfügen, die den Entscheidungsprozess erleichtern könnten (Sniderman et al. 1991, S. 93-119; Fazio et al. 1992). Demnach wäre zu erwarten, dass ein größerer Anteil der Befragten mit „weiß nicht“ antworten würde, um den enormen kognitiven Aufwand nicht auf sich 
nehmen zu müssen. Liegen die Parteinamen hingehen visuell vor, erleichtert dies das Treffen einer Wahlentscheidung erheblich.

Um zu überprüfen, ob die Zunahme unentschlossener Wähler durch den Wechsel des Erhebungsmodus erklärt werden kann, stehen Daten aus einem natürlichen Experiment zur Verfügung. Das „Politbarometer“ ist eine sozialwissenschaftliche Trenduntersuchung der „Forschungsgruppe Wahlen e. V.“, in deren Rahmen seit 1977 fast jeden Monat repräsentative Querschnittsuntersuchungen zu politischen Einstellungen und Verhaltensweisen der Deutschen durchgeführt werden. Dabei wurden bis August 1988 alle Untersuchungen face-to-face durchgeführt und die Wahlabsicht dementsprechend gestützt abgefragt. Seit September 1988 erfolgen alle Interviews jedoch telefonisch, und die Wahlabsicht wird seitdem über eine offene Frage erhoben. Dieselbe Umstellung des Erhebungsmodus erfolgte in Ostdeutschland im Juli 1995.

\section{Abbildung 4: Monatliche Anteile unentschlossener Wähler im Politbarometer, vor der Umstellung auf CATI und danach}

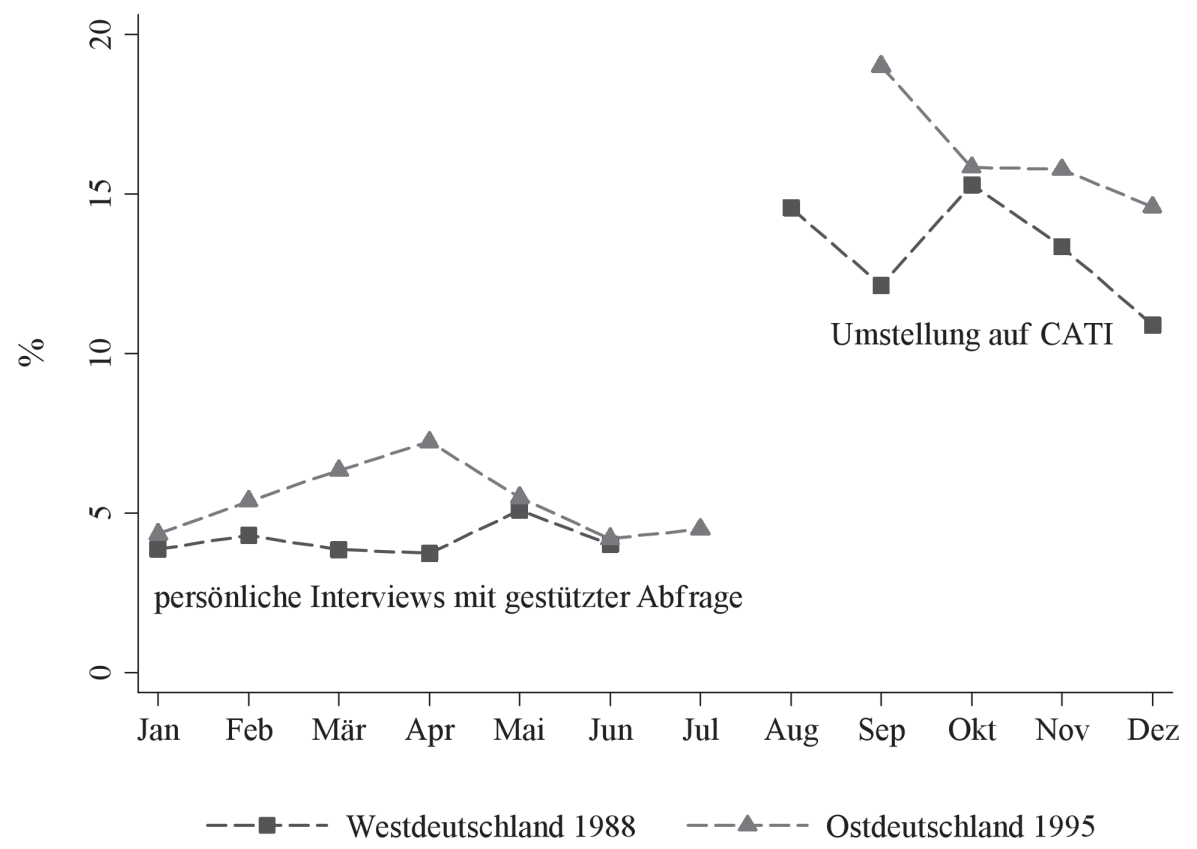

Daten: Berger et al. 1988; Berger et al. 1995.

Falls die Veränderung des Erhebungsmodus tatsächlich die Hauptursache für die starke Zunahme von Unentschlossenheit darstellt, sollte man in PolitbarometerZeitreihen eine Zunahme von unentschlossenen Wählern im August 1988 in Westdeutschland sowie im September 1995 in Ostdeutschland beobachten können. Genau das lässt sich feststellen (vgl. Abbildung 4): Nach der Umstellung auf CATI erhöhte sich der Anteil der unentschlossenen Wähler sowohl in West- als 
auch in Ostdeutschland schlagartig um etwa 10 Prozentpunkte. Eine offene Frage nach der Wahlintention ruft somit deutlich mehr Unentschlossenheit hervor als eine gestützte Abfrage unter Vorlage eines Listenhefts (vgl. auch Jung 1990, S. 408; Erhardt 1998, S. 45, 81).

Auch mit anderen Daten lässt sich nachweisen, dass eine nicht-gestützte Abfrage der Wahlabsicht deutlich mehr Unentschlossenheit hervorruft. In der Vorwahl-Querschnittsstudie, die im Rahmen der „German Longitudinal Election Study“ (GLES) vor der Bundestagswahl 2009 face-to-face und mit gestützter Abfrage der Wahlabsicht durchgeführt wurde, liegt der Anteil der unentschlossenen Wähler bei knapp 15 Prozent, während der entsprechende Anteil in der etwa zeitgleich durchgeführten RCS-Studie bei etwa 25 Prozent liegt. Auch hier beträgt der Unterschied zwischen gestützter und nicht-gestützter Abfrage 10 Prozentpunkte.

Was bedeutet dieser Befund für den in Abbildung 3 festgestellten Anstieg später Wahlentscheidungen? Es sei daran erinnert, dass der festgestellte Niveauunterschied im Anteil der noch nicht endgültig entschiedenen Wähler zwischen den älteren und jüngeren Wahlstudien ausschließlich durch eine dramatische Zunahme des Anteils unentschlossener Wähler zustande kam. Es konnte weiterhin gezeigt werden, dass etwa 10 Prozentpunkte in der Zunahme der UnentschlossenenAnteile durch den Modus-Wechsel von face-to-face auf CATI erklärt werden können. Es ist daher anzunehmen, dass ein großer Teil der unentschlossenen Wähler, hätte man die RCS-Studien 2005 und 2009 face-to-face mit gestützter Abfrage der Wahlabsicht durchgeführt, eine Partei genannt hätte. Davon hätte ein Teil von ihnen eine Partei genannt, die mit ihrer späteren Parteiwahl übereingestimmt hätte, während ein anderer Teil die Parteipräferenzen zwischen Vorwahlinterview und der Wahl nochmals geändert hätte. Es ist somit wahrscheinlich, dass in einem face-to-face-Interview der Anteil der unentschlossenen Wähler um etwa 10 Prozentpunkte niedriger ausfiele, der Anteil der Präferenzwechsler im Gegenzug jedoch um ein paar Prozentpunkte anstiege. Der Verfasser dieser Arbeit schätzt daher, dass der in Abbildung 3 dargestellte Anstieg später Wahlentscheidungen um etwa 5 bis 8 Prozentpunkte niedriger ausgefallen wäre, wenn kein Moduswechsel stattgefunden hätte.

Wenn man die Anteilswerte für die Jahre 2005 und 2009 in Abbildung 3 um diese Größenordnung reduzieren würde, könnte man zwar noch immer von einer Zunahme der noch nicht entschlossenen Wähler sprechen. Diese Zunahme fiele aber moderater aus, als man aufgrund der auf der Rückerinnerungsmethode basierenden Ergebnisse in Abbildung 1 hätte vermuten können. Dies deutet darauf hin, dass die Rückerinnerungsmethode Validitätsdefizite aufweist.

\section{Eine Validierungsanalyse auf individueller Ebene}

Um die Validität der Rückerinnerungsmethode auf individueller Ebene zu beurteilen, sind Daten erforderlich, mit denen die Entscheidungszeitpunkte der Befragten sowohl über die Rückerinnerungs- als auch über die Panelmethode operationalisiert und somit verglichen werden können. Sechs Panelstudien (Klingemann u. Pappi 1969; Berger et al. 1972; Berger et al. 1976; Kaase et al. 1990; Schmitt- 
Beck u. Faas 2005; Rattinger et al. 2009b) erfüllen diese Bedingung und werden im Folgenden verwendet. Aus Platzgründen werden hier nur die Ergebnisse zweier charakteristischer Studien (1972 und 2009) detailliert dargestellt, während die Ergebnisse der anderen Panelstudien in einem Online-Appendix veröffentlicht werden.

Ein Vergleich von Rückerinnerungs- und Panelmethode erfordert, dass die in der Rückerinnerungsmethode eher vage formulierten Antwortvorgaben in konkrete Zeiträume übersetzt werden. Welchen Zeitraum umfasst der Begriff „letzte Monate“ und welcher Zeitraum ist mit „letzte Wochen“ gemeint? Da Personen diese Begriffe vermutlich unterschiedlich interpretieren, wurden hier eher großzügige Übersetzungen vorgenommen, welche die Wahrscheinlichkeit reduzieren, dass die Antworten der Probanden auf die Rückerinnerungsfrage als inkonsistent mit der Panelmethode eingestuft werden. Für Personen, die nach eigenen Angaben ihre Entscheidung „in den letzten Tagen“ trafen, wurde angenommen, dass sie damit die letzten sieben Tage vor der Wahl meinten. Der Begriff „letzte Wochen“ wurde übersetzt mit eine bis fünf Wochen vor der Wahl, „letzte Monate“ mit sechs bis zwanzig Wochen vor der Wahl und „stand seit langem fest" bedeutet demnach, dass die Entscheidung über 20 Wochen vor der Wahl getroffen wurde.

Bei der Bestimmung der Entscheidungszeiträume über die Panelmethode wurden hier, im Gegensatz zu den Analysen im vorherigen Abschnitt, alle zur Verfügung stehenden Informationen aus den Panelwellen verwendet. Wenn also eine Person in der ersten Welle eine mit der späteren Parteiwahl konsistente Wahlabsicht nannte, diese in der zweiten Welle jedoch revidierte, so gilt diese Person in der ersten Welle als noch nicht entschieden. Darüber hinaus wurde auch der genaue Tag, an dem das Vorwahlinterview durchgeführt wurde, bei der Berechnung des Entscheidungszeitraums berücksichtigt. Diese Information war vor allem dann hilfreich, wenn sich der Erhebungszeitraum einer Vorwahl-Panelwelle über mehrere Wochen erstreckte und auf diese Weise die Schwellenwerte überschritt, welche die „letzten Monate“, „letzten Wochen“ und „letzten Tage“ voneinander abgrenzen.

Trotz des Versuchs, die Antwortvorgaben der Rückerinnerungsmethode möglichst exakt nachzuzeichnen, sind die mit der Panelmethode gebildeten Entscheidungszeiträume notwendigerweise unpräzise. So etwa reichen die Panelwellen meist nicht so lange in die Zeit zurück, dass sich die Angabe, die Entscheidung stehe „seit langem“ fest, überprüfen lässt (Ausnahme ist die Wahlstudie 1990). Wenn beispielsweise das erste Interview acht Wochen vor der Wahl stattfand und die Person in allen Vorwahlinterviews jene Partei als Wahlabsicht nannte, die sie später auch wählte, dann kann sie sich entweder in den „letzten Monaten“ entschieden haben oder bereits „seit langem“ entschlossen gewesen sein. Der Entscheidungszeitraum lässt sich anhand der gegebenen Daten nicht näher eingrenzen. Das ist der Grund dafür, weshalb vielen Befragten mehrere theoretisch mögliche Entscheidungszeiträume zugewiesen wurden, wie anhand der nachfolgenden Tabellen ersichtlich wird. 
Tabelle 2: Vergleich des Zeitpunkts der Wahlentscheidung nach der Panelmethode und nach der Rückerinnerungsmethode, Wahlstudie 1972 (Prozentanteile)

\begin{tabular}{|c|c|c|c|c|}
\hline \multirow[b]{2}{*}{ Rückerinnerung } & \multicolumn{3}{|c|}{ Panelmethode } & \multirow[b]{2}{*}{$\%$ Gesamt } \\
\hline & (1) bis (2) & (2) bis (3) & (3) & \\
\hline (1) Seit langem & $\begin{array}{c}88,2 \\
(61,6)\end{array}$ & $\begin{array}{l}63,6 \\
(6,1) \\
\end{array}$ & $\begin{array}{c}64,5 \\
(13,2)\end{array}$ & 80,9 \\
\hline (2) Letzte Monate & $\begin{array}{c}8,0 \\
(5,6) \\
\end{array}$ & $\begin{array}{l}16,8 \\
(1,6)\end{array}$ & $\begin{array}{l}14,9 \\
(3,1) \\
\end{array}$ & 10,3 \\
\hline (3) Letzte Wochen & $\begin{array}{c}3,9 \\
(2,7) \\
\end{array}$ & $\begin{array}{l}19,6 \\
(1,9) \\
\end{array}$ & $\begin{array}{r}20,6 \\
(4,2) \\
\end{array}$ & 8,8 \\
\hline$\%$ Gesamt & $\begin{array}{c}100,0 \\
(69,9) \\
777\end{array}$ & $\begin{array}{c}100,0 \\
(9,6) \\
107\end{array}$ & $\begin{array}{c}100,0 \\
(20,5) \\
287\end{array}$ & $\begin{array}{l}100,0 \\
1112\end{array}$ \\
\hline
\end{tabular}

Erläuterung: Werte in der ersten Zeile sind Spaltenprozente, Werte in Klammem sind Zellenprozente. (1) bis (2) bedeutet, dass der Befragte sich im Zeitraum zwischen "seit langem" und "letzten Wochen“ entschieden haben muss; eine nähere Eingrenzung des Entscheidungszeitraums ist anhand der Panelmethode nicht möglich. Aufgrund von Rundungsfehlern addieren sich die Prozentwerte nicht immer auf 100 Prozent. Interpretationsbeispiel: 88,2 Prozent der Personen, die sich nach der Panelmethode "seit langem“ oder „in den letzten Monaten“ entschieden hatten, gaben bei der Rückerinnerung an, „seit langem“ entschlossen gewesen zu sein. Dies entspricht 61,6 Prozent der Gesamtstichprobe. Daten: Berger et al. 1972.

Die aus den beiden Messmethoden resultierenden Entscheidungszeitpunkte für die Wahl 1972 sind in Tabelle 2 in Form einer Kreuztabelle dargestellt. Dabei repräsentieren die Zeilen die Entscheidungszeitpunkte gemäß der Rückerinnerungsmethode und die Spalten jene gemäß der Panelmethode. Auf den ersten Blick scheinen die Ergebnisse die Validität der Rückerinnerungsmethode zu stützen, denn etwa 75 Prozent der Antworten auf die Rückerinnerungsfrage stehen nicht im Widerspruch zu den Ergebnissen der Panelmethode (weiß hinterlegte Zellen). Der größte Teil (62 Prozent) davon sind Befragte, die angaben, bereits „seit langer Zeit" entschieden gewesen zu sein und gleichzeitig, mit dieser Angabe kompatibel, stabile Wahlintentionen in den Vorwahlbefragungen aufwiesen. Weitere 3 Prozent der Wähler gaben an, sie hätten sich erst in den letzten Wochen vor der Wahl auf eine Partei festgelegt, gelten nach der Panelmethode aber als bereits schon länger entschieden (hellgrau hinterlegte Zellen). Dieser scheinbare Widerspruch ist nicht notwendigerweise als „Fehler“ zu werten, weil es sein kann, dass bei diesen Befragten zwischen zwei Panelwellen unbeobachtete Präferenzveränderungen stattfanden. Stabile Wahlabsichten an den einzelnen Befragungszeitpunkten implizieren nicht notwendigerweise, dass die Präferenzen tatsächlich über den gesamten Zeitraum stabil waren.

Es gibt somit nur ein einziges Antwortmuster, welches eindeutig als Indikator für fehlende Validität der Rückerinnerungsmethode gedeutet werden kann: wenn eine Person angab, bereits „seit langem“ entschlossen gewesen zu sein, obwohl aus den Vorwahldaten hervorgeht, dass ihre Entscheidung kurz vor der Wahl 
noch nicht feststand (dunkelgrau hinterlegte Zellen). In dieser Hinsicht schneidet die Rückerinnerungsmethode schlecht ab: 64 Prozent der Befragten, die ihre Wahlentscheidung nach der Panelmethode entweder in den letzten Monaten oder letzten Wochen vor der Wahl trafen, betrachteten sich als „seit langem“ entschieden. Ebenso äußerten sich 65 Prozent der Befragten, die ihre Entscheidung nach der Panelmethode erst in den letzten Wochen vor der Wahl getroffen haben konnten, ihre Entscheidung hätte schon seit langem festgestanden. Das heißt, fast zwei Drittel aller sich sehr spät entscheidenden Wähler werden durch die Rückerinnerungsmethode fälschlicherweise als „frühe“ Entscheider klassifiziert.

Die massive Verzerrung in den Messwerten der Rückerinnerungsfrage führt zu unterschiedlichen Randverteilungen der beiden Messmethoden: Während nach der Rückerinnerungsmethode 9 Prozent angaben, sie hätten ihre Wahlentscheidung in den letzten Wochen vor der Wahl getroffen, sind es nach der Panelmethode 20 bis 30 Prozent. Nach der Rückerinnerungsmethode bekundeten 81 Prozent, sie hätten ihre Wahlentscheidung „seit langem“ getroffen, während es nach der Panelmethode 70 Prozent waren, die ihre Entscheidung „seit langem“ oder in den letzten Monaten trafen.

Die (hier nicht dargestellten) Validierungsanalysen mit den Daten aus den Jahren 1969, 1976 und 1990 liefern ein sehr ähnliches Bild. Insgesamt sind jeweils etwa drei Viertel der beiden Messwerte konsistent, was eigentlich ein relativ gutes Ergebnis darstellt und somit oberflächlich für die Validität der Rückerinnerungsmethode spricht. Dieses gute Ergebnis kommt jedoch allein dadurch zustande, dass die Rückerinnerungsmethode eine starke Verzerrung zugunsten der Messung „früher" Wahlentscheidungen aufweist. Da die meisten Befragten auch nach der Panelmethode „frühe“ Wahlentscheidungen trafen, wurden diese Befragten korrekt klassifiziert. Das Problem besteht jedoch darin, dass die zahlenmäßig kleinere Gruppe der nach der Panelmethode eindeutig als „späte“ Entscheider gekennzeichneten Wähler mehrheitlich fälschlicherweise eine „frühe“ Entscheidung bekundeten. Die Verwendung der Rückerinnerungsdaten führte somit in den frühen Wahlstudien zu einer deutlichen Überschätzung des Anteils der „seit langem“ feststehenden Wahlentscheidungen. 
Tabelle 3: Vergleich des Zeitpunkts der Wahlentscheidung nach der Panelmethode und nach der Rückerinnerungsmethode, RCS-Studie 2009 (Prozentanteile)

\begin{tabular}{|c|c|c|c|c|c|c|}
\hline \multirow[b]{2}{*}{ Rückerinnerung } & \multicolumn{5}{|c|}{ Panelmethode } & \multirow[b]{2}{*}{$\%$ Gesamt } \\
\hline & (1) bis (2) & (1) bis (3) & (1) bis (5) & (3) bis (5) & (4) bis (5) & \\
\hline (1) Seit langem & $\begin{array}{c}58,9 \\
(11,9)\end{array}$ & $\begin{array}{c}54,0 \\
(16,8)\end{array}$ & $\begin{array}{l}48,2 \\
(4,5)\end{array}$ & $\begin{array}{l}26,0 \\
(9,1)\end{array}$ & $\begin{array}{l}25,8 \\
(1,2)\end{array}$ & 43,4 \\
\hline (2) Letzte Monate & $\begin{array}{l}16,2 \\
(3,3)\end{array}$ & $\begin{array}{l}18,7 \\
(5,8)\end{array}$ & $\begin{array}{l}16,1 \\
(1,5)\end{array}$ & $\begin{array}{l}12,5 \\
(4,3)\end{array}$ & $\begin{array}{c}10,8 \\
(0,5)\end{array}$ & 15,4 \\
\hline (3) Letzte Wochen & $\begin{array}{c}13,8 \\
(2,8)\end{array}$ & $\begin{array}{c}14,8 \\
(4,6)\end{array}$ & $\begin{array}{c}19,7 \\
(1,8)\end{array}$ & $\begin{array}{c}22,1 \\
(7,7)\end{array}$ & $\begin{array}{c}20,4 \\
(1,0)\end{array}$ & 17,9 \\
\hline (4) Letzte Tage & $\begin{array}{c}7,3 \\
(2,8)\end{array}$ & $\begin{array}{c}8,5 \\
(2,6)\end{array}$ & $\begin{array}{l}11,5 \\
(1,1)\end{array}$ & $\begin{array}{l}26,0 \\
(9,1)\end{array}$ & $\begin{array}{l}22,2 \\
(1,1)\end{array}$ & 15,3 \\
\hline (5) Wahltag selbst & $\begin{array}{c}3,8 \\
(0,8) \\
\end{array}$ & $\begin{array}{c}4,0 \\
(1,2)\end{array}$ & $\begin{array}{c}4,6 \\
(0,4)\end{array}$ & $\begin{array}{l}13,3 \\
(4,6)\end{array}$ & $\begin{array}{l}21,0 \\
(1,0)\end{array}$ & 8,1 \\
\hline$\%$ Gesamt & $\begin{array}{l}100,0 \\
(20,0)\end{array}$ & $\begin{array}{l}100,0 \\
(31,1)\end{array}$ & $\begin{array}{c}100,0 \\
(9,3)\end{array}$ & $\begin{array}{l}100,0 \\
(34,7)\end{array}$ & $\begin{array}{c}100,0 \\
(4,7)\end{array}$ & 100,0 \\
\hline$n$ & 711 & 1100 & 330 & 1229 & 167 & 3537 \\
\hline
\end{tabular}

Erläuterung: vgl. Tabelle 2.

Daten: Rattinger et al. 2009b.

Anders ist dies bei den RCS-Studien 2005 und 2009 (dargestellt in Tabelle 3 sind nur die Daten aus 2009): Hier zeigt sich, dass die Rückerinnerungsmethode besser zwischen frühen und späten Wahlentscheidungen differenziert. Gemessen an allen Befragten in 2005 und 2009, die ihre Wahlentscheidung gemäß der Panelmethode erst in den letzten Wochen oder letzten Tagen trafen, gaben nur noch 36 bzw. 38 Prozent fälschlicherweise an, dass ihre Wahlentscheidung bereits in den letzten Monaten oder noch länger festgestanden hätte. 1976 lag dieser Wert noch doppelt so hoch. Aus einem Grund, der nicht offensichtlich wird, passten sich die Antworten auf die Rückerinnerungsfrage im Zeitverlauf den tatsächlichen Entscheidungszeiträumen an.

Um ausschließen zu können, dass diese plötzliche Anpassung der Ergebnisse der Rückerinnerungsmethode an die Ergebnisse der Panelmethode ein Spezifikum von telefonisch durchgeführten, zweiwelligen RCS-Studien darstellt, wurde mit dem online durchgeführten GLES-Wahlkampfpanel zur Bundestagswahl 2009 (Rattinger et al. 2009c) noch ein zusätzlicher Datensatz hinzugezogen. Das Wahlkampfpanel wurde im vorangegangenen Abschnitt, als es um die Entwicklung des Entscheidungszeitpunkts im Zeitverlauf ging, ausgeklammert, da die Ergebnisse der Online-Umfrage nicht repräsentativ für das Gesamtelektorat sind. Zum Zwecke der Validierung einer Messmethode ist die Repräsentativität der Befragten aber kein entscheidendes Kriterium. Das Wahlkampfpanel verfügt über sieben Befragungszeitpunkte (sechs vor der Wahl, eine Nachwahlerhebung). Die Wahlabsicht wurde gestützt abgefragt, allerdings mit expliziter Vorgabe einer „weiß nicht"-Option. Die Anteile unentschlossener Wähler sind mit 12 Prozent (Welle 6) bis 23 Prozent (Welle 1) wesentlich geringer als in den beiden RCS-Studien. 
Noch eindrucksvoller als bei den RCS-Studien zeigt sich in der WahlkampfPanelstudie, dass sich die Rückerinnerungsmethode mittlerweile zu einem validen Indikator zur Messung des Entscheidungszeitpunkts entwickelt hat (Ergebnisse sind im Online-Appendix abgebildet). Nur noch 26 Prozent aller Befragten, die sich nach der Panelmethode objektiv erst innerhalb der letzten Wochen für eine Partei entschieden, gaben bei der Rückerinnerungsfrage fälschlicherweise an, sie hätten ihre Wahlentscheidung bereits in den letzten Monaten oder noch früher getroffen.

\section{Tabelle 4: Übersicht über Fehlerquoten bei Validierungsanalysen der deutschen Wahlstudien}

\begin{tabular}{lcc}
\hline Studie & $\begin{array}{c}\text { Anteil der „Frühentscheider“ nach der R-Methode an } \\
\text { allen ,Spätentscheidern “ nach der P-Methode } \\
\text { (in \%) }\end{array}$ & $\begin{array}{c}\text { Fehleranteil } \\
\text { insgesamt } \\
\text { (in \%) }\end{array}$ \\
\cline { 3 - 4 } BTW 1969 & 86,2 & 21,6 \\
BTW 1972 & 74,4 & 22,4 \\
BTW 1976 & 75,3 & 23,8 \\
BTW 1990 & 65,5 & 15,4 \\
BTW 2005 & 39,2 & 17,2 \\
BTW 2009 (RCS) & 40,9 & 16,1 \\
BTW 2009 (WKP) & 37,1 & 15,0 \\
\hline
\end{tabular}

Erläuterung: $\mathrm{BTW}=$ Bundestagswahl; $\mathrm{WKP}=$ Wahlkampfpanel; $\mathrm{R}=$ Rückerinnerungsmethode; $\mathrm{P}=$ Panelmethode.

Daten: Klingemann u. Pappi 1969; Berger et al. 1972; Berger et al. 1976; Kaase et al. 1990; Schmitt-Beck u. Faas 2005; Rattinger et al. 2009b, 2009c.

In Tabelle 4 sind die wichtigsten Ergebnisse der Validierungsanalysen zusammengefasst. In der ersten Spalte sind die Fehlerraten in Form von Spaltenprozenten dargestellt, in der zweiten Spalte in der Form von Zellenprozenten. Die Spaltenprozente machen deutlich, dass die Rückerinnerungsmethode in den frühen Wahlstudien nicht in der Lage war, späte Wahlentscheidungen korrekt zu messen. Bei der Studie zur Bundestagswahl 1969 äußerten 86 Prozent aller Befragten, die zum Zeitpunkt ihres eine bis zwei Wochen vor der Wahl durchgeführten Vorwahlinterviews objektiv noch nicht entschlossen waren, dass sie ihre Entscheidung bereits „seit langem“ oder „in den letzten Monaten“ getroffen hätten. Dieser Fehleranteil reduziert sich im Zeitverlauf enorm und erreicht im Wahlkampfpanel 2009 seinen aktuellen Tiefststand. Man könnte von einer „Normalisierung“ der Messqualität sprechen: Die Rückerinnerungsmethode gewinnt graduell an Validität, auch wenn ein Fehleranteil von 37 Prozent noch immer nicht zufriedenstellend ist.

Wenn man den Fehler nicht auf die Spätentscheider, sondern auf die Gesamtstichprobe prozentuiert, erhält man die Fehlerraten in Spalte 2. Diese gehen im Zeitverlauf nur leicht zurück, von einem Maximum von 24 Prozent im Jahr 1976 auf bis zu 15 Prozent im Wahlkampfpanel 2009. Der Grund für diesen nur moderaten Rückgang ist in zwei gegenläufigen Trends zu sehen, die sich tendenziell gegenseitig neutralisieren: Die Zunahme der Validität der Rückerinnerungs- 
frage geht einher mit einer „realen“ Zunahme später Wahlentscheidungen, die sich auch in den Paneldaten manifestiert. Somit erhöhte sich im Zeitverlauf die Anzahl der Befragten, die potenziell durch die Rückerinnerungsmethode fehlklassifiziert werden kann, während gleichzeitig die Tendenz zur Fehlklassifikation abnahm.

\section{Diskussion}

Warum bestand früher eine beträchtliche systematische Verzerrung in den Messwerten der Rückerinnerungsfrage, und warum nimmt diese Verzerrung im Zeitverlauf kontinuierlich ab? Der Verfasser dieses Aufsatzes stellte diese Frage in mehreren Vorträgen zur Diskussion und sammelte auf diese Weise verschiedene Erklärungsansätze. Obwohl aus meiner Sicht keiner von ihnen völlig überzeugt, seien sie hier kurz diskutiert.

Soziale Erwünschtheit. Die mit Abstand am häufigsten geäußerte Vermutung lautet, dass "frühe“ Wahlentscheidungen in der Vergangenheit ein sozial erwünschtes Verhalten dargestellt hätten. Demnach habe bis in die Siebzigerjahre eine stark ausgeprägte soziale Norm bestanden, sich klar zu einer Partei zu bekennen. Personen, die diese Norm internalisiert hatten, seien in einer Interviewsituation bestrebt gewesen, keinerlei Zweifel an der Überzeugtheit von ihrer eigenen Entscheidung aufkommen zu lassen und hätten daher, selbst wenn es nicht der Wahrheit entsprochen habe, eine bereits „seit langem“ feststehende Entscheidung angegeben. Im Zeitverlauf habe sich diese Norm dann abgeschwächt. Möglicherweise habe sich sogar eine neue, gegenteilige Norm etabliert, nämlich der Wunsch, sich als möglichst unabhängigen und sorgfältigen Entscheider zu präsentieren. Die Folge sei gewesen, dass sich die „Spätentscheider“ nunmehr zu ihrer späten Entscheidung bekannt oder sogar späte Wahlentscheidungen vorgetäuscht hätten.

In Unterstützung dieser These des sozialen Drucks könnte ins Feld geführt werden, dass auch Noelle-Neumann (2001) in den Sechziger- und Siebzigerjahren einen starken gesellschaftlichen Konformitätszwang behauptete. Folgt man ihrer Theorie der "Schweigespirale“, schlügen sich Befragte in Umfragen tendenziell auf die Seite jener Partei, die in der Gesellschaft als mehrheitlich favorisiert wahrgenommen werde. Noelle-Neumann zufolge hätten damals SPD-Anhänger über die Meinungsführerschaft verfügt, gegen die sich die stille konservative Mehrheit verbal nicht zu widersetzen gewagt habe. Dieser Umstand wurde als Erklärung dafür angesehen, dass der SPD-Anteil in politischen Umfragen stark überschätzt wurde. Auch wenn es keinen direkten Zusammenhang mit der Messung des Entscheidungszeitpunkts gibt, kann das Entstehen dieser Theorie (und deren abnehmende Popularität im Zeitverlauf) zumindest als Indiz dafür gewertet werden, dass der Befolgung sozialer Normen früher eine größere Bedeutung zukam als heutzutage. Der Verfasser dieser Arbeit ist persönlich zwar eher skeptisch, dass der Entscheidungszeitpunkt ein normativ derart aufgeladenes Thema gewesen sein könnte; gleichfalls kann die Möglichkeit nicht ausgeschlossen werden.

Diskontinuitäten im Messinstrument. Folgt man diesem Erklärungsversuch, dann sind für die Zunahme später Wahlentscheidungen nach der Rückerinnerungsmethode hauptsächlich Veränderungen im Messinstrument verantwort- 
lich. Früher wurde die Frage nach dem Entscheidungszeitpunkt mit deutlich weniger Antwortvorgaben gestellt und die Befragten mussten lediglich zwischen den Optionen „seit langem“, „letzte Monate“ und „letzte Wochen“ auswählen. Über die Jahre kamen mit den Vorgaben „letzte Tage“ und „am Wahltag selbst“ weitere Antwortvorgaben hinzu. Krosnick (1999) argumentiert, dass eine orale Präsentation einer langen Reihe von Antwortoptionen eher dazu führe, dass die letzte Antwortvorgabe gewählt werde, weil der genaue Wortlaut im Gegensatz zur ersten Antwortvorgabe noch im Gedächtnis sei (ein sogenannter recency-Effekt). Weil bei der Rückerinnerungsfrage die späten Zeiträume am Ende der Frage präsentiert würden, könne es sein, dass die Zunahme später Wahlentscheidungen auf einem der zunehmenden Komplexität der Fragestellung geschuldeten recencyEffekt zurückzuführen sei.

Dieser Erklärungsversuch kann jedoch eindeutig verworfen werden. Aus dem Vergleich der telefonisch durchgeführten RCS-Studie 2009 mit dem online durchgeführten Wahlkampfpanel 2009 geht hervor, dass eine visuelle Präsentation der Antwortvorgaben (im Vergleich zur oralen Präsentation) nichts an der Verteilung der Antwortnennungen ändert. Ansonsten hat sich am Messinstrument nichts verändert, was theoretisch einen Rückgang der Fehlerraten erklären könnte.

Unentschlossene Wähler. Die beiden letzten Erklärungsversuche unterscheiden sich insofern von den vorhergehenden, als sie die Validitätsdefizite und Ursache für die variierenden Fehlerraten nicht in der Rückerinnerungs-, sondern in der Panelmethode sehen. Bislang wurde davon ausgegangen, dass die Panelmethode fehlerfrei in der Lage wäre, noch nicht endgültig entschiedene Wähler zu identifizieren. Allerdings wurde bereits in Abschnitt 4 dieses Aufsatzes demonstriert, dass die Paneldaten unterschiedliche Anteile an unentschlossenen Wählern produzieren, je nachdem welche Erhebungsmethode verwendet wird. Kann die Umstellung von face-to-face auf CATI den Rückgang der Fehleranteile erklären?

Auch mit diesem Erklärungsansatz lässt sich das Rätsel nicht lösen. Wie aus Tabelle 4 hervorgeht, gingen die Fehlerraten bereits in den Wahlstudien zwischen 1969 bis 1990 zurück, die allesamt face-to-face durchgeführt wurden. Die Möglichkeit, dass der Erhebungsmodus ausschlaggebend ist, wird auch durch die Tatsache entkräftet, dass man mit der ungestützten Abfrage der Wahlabsicht im Online-Wahlkampfpanel 2009 zu ähnlichen Ergebnissen gelangt wie bei der ungestützten Abfrage in der (CATI-) RCS-Studie 2009. Abgesehen davon lässt sich bereits logisch ableiten, dass die unter CATI-Bedingungen erhöhte Tendenz, sich als unentschlossen zu betrachten, keinen Einfluss auf die Höhe der gemessenen Fehlerraten haben konnte. Wenn sich jemand unter CATI-Bedingungen als unentschlossen einstuft, obwohl dieselbe Person in einem face-to-face-Interview eine Parteipräferenz genannt hat, dann mag die CATI-Antwort vielleicht eher den Tatsachen entsprechen. Die durch die Umstellung auf CATI verbesserte Messqualität würde dann daraus resultieren, dass Wähler, die in der Vor-CATI-Zeit durch die Panelmethode fälschlicherweise als Frühentscheider klassifiziert worden wären, nun korrekt als Spätentscheider identifiziert werden würden. Doch unter der Annahme, dass die Rückerinnerungsmethode valide ist, beobachten wir genau das gegenteilige Muster: Wähler, die in der Vor-CATI-Zeit durch die Panelmethode fälschlicherweise als Spätentscheider klassifiziert worden waren, wurden seit der 
Umstellung auf CATI nunmehr korrekt als Frühentscheider identifiziert. Wenn also tatsächlich Unzulänglichkeiten der Panelmethode für die hohen Fehleranteile in den frühen Wahlstudien verantwortlich sein sollen, müsste man nachweisen, dass die bei der Panelmethode vorgenommene Identifikation von späten Wahlentscheidungen früher stärker fehlerbehaftet war als heute. Das führt uns zum letzten Punkt.

Messfehler bei der Erhebung der Parteiwabl. Nach der Panelmethode wird ein Befragter als „später“ Entscheider klassifiziert, wenn dieser in einem Interview kurz vor der Wahl a) sich noch nicht zu einer Wahlteilnahme entschlossen hat, b) als Wahlintention eine Partei nannte, die nicht mit der später gewählten Partei übereinstimmte oder c) noch unentschlossen war. Die Bedingungen a) und c) werden hier ignoriert, da deren Anteilswerte in den frühen Wahlstudien quantitativ nicht so stark ins Gewicht fielen, als dass deren fehlerhafte Messung die enormen Inkonsistenzen erklären könnte. Somit bleibt Bedingung b) übrig. Die Panelmethode betrachtet eine Person kurz vor der Wahl als noch nicht endgültig entschieden, wenn sie beispielsweise eine Woche vor der Wahl die SPD zu wählen beabsichtigte, in der Nachwahlwelle jedoch angab, die CDU gewählt zu haben. Nun kann es sein, dass einer dieser beiden Angaben falsch ist. Die Person wählte tatsächlich die SPD oder wies bereits im Vorwahlinterview eine CDU-Wahlintention auf. Die Person muss nicht unbedingt gelogen haben, es könnte sich auch um Codierungsfehler handeln. Messfehler in der Erhebung der Wahlintention und Wahlverhalten könnten erklären, warum die Panelmethode mehr Spätentscheider produziert als die Rückerinnerungsmethode.

Es wäre unrealistisch anzunehmen, dass bei der Erhebung der Parteiwahl keine Messfehler passieren. Allerdings besteht hier der erklärungsbedürftige Umstand darin, weshalb sich der Messfehler kontinuierlich verringert haben sollte. Wenn man berücksichtigt, dass sich das politische Angebot im Zeitverlauf ausdifferenzierte und mittlerweile deutlich mehr Parteien auf dem Fragebogen stehen als noch vor 40 Jahren, müsste man eher davon ausgehen, dass sich die Chancen für Messfehler erhöhten. Es gibt ebenso keinen theoretischen Grund zu der Annahme, dass die Befragten heutzutage ehrlicher sind als früher, abgesehen von der bereits diskutierten Möglichkeit, dass die Antworten früher eventuell stärker Konformitätszwänge reflektierten.

Letztlich wird es nicht möglich sein, eine Erklärung zu finden. Was die ganze Sachlage noch rätselhafter macht, ist die Tatsache, dass die anhand der Rückerinnerungsmethode festgestellte Zunahme später Wahlentscheidung kein ausschließlich deutsches Phänomen ist, sondern dass sich dieser Trend gleichzeitig in zahlreichen westlichen Demokratien abspielt (wenn auch nicht in demselben Ausmaß; vgl. z. B. Dalton et al. 2000; McAllister 2002, Plasser et al. 2003; Irwin u. Van Holsteyn 2008). Bislang ist man davon ausgegangen, dass die Zunahme später Wahlentscheidungen in all diesen Ländern eine inhaltliche Ursache habe, namentlich die Abnahme langfristig stabiler Parteibindungen. Wäre es möglich, dass sich die Zunahme später Wahlentscheidung auch in anderen Ländern zumindest teilweise als methodisches Artefakt herausstellt? Es könnte lohnenswert sein, dies in Zukunft näher zu beleuchten. 


\section{Schlussfolgerung}

Der Zeitpunkt der Wahlentscheidung ist ein wichtiger Indikator für das Wirkungspotenzial von Wahlkämpfen. Wenn sich alle Wähler bereits lange vor der Wahl entscheiden würden, wären Wahlkämpfe offensichtlich irrelevant. Umgekehrt, wenn alle Wähler während des Wahlkampfes ihre Entscheidung träfen, bestünde zumindest die Möglichkeit, dass Wähler bei ihrer Entscheidungsfindung Wahlkampfinformationen berücksichtigen würden. Von hoher Relevanz sind daher Zeitreihen, die eine starke Zunahme von im Wahlkampf getroffenen Wahlentscheidungen belegen. Vor allem die Zunahme der Spätentscheider-Anteile bei den Bundestagswahlen in den Jahren 2005 und 2009 scheinen darauf hinzudeuten, dass die Wahlkampfzeit für den Ausgang von Wahlen rapide an Bedeutung gewinnt.

Die in bisherigen Untersuchungen festgestellte Zunahme später Wahlentscheidungen (Dalton et al. 2000; McAllister 2002; Plasser et al. 2003; Irwin u. Van Holsteyn 2008, Plischke u. Bergmann 2012) basierte auf Daten, die durch retrospektive Selbsteinschätzungen gewonnen wurden. Aus der Wechselwahlforschung und aus anderen sozialwissenschaftlichen Forschungsfeldern ist bekannt, dass die Rückerinnerungen von Befragten mit Vorsicht zu genießen sind. Sind Wähler tatsächlich dazu in der Lage, einzuschätzen, wann sie ihre endgültige Entscheidung trafen? Dieser Frage wurde in diesem Beitrag nachgegangen, indem die Antworten auf die Rückerinnerungsfrage mit Verhaltensintentionen in Vorwahlbefragungen verglichen wurden.

Die Ergebnisse sind überraschend und weisen eine Vielzahl von Implikationen auf. Als erstes bleibt festzuhalten, dass die Angaben, die Wähler bei der Rückerinnerungsfrage machen, in der Regel mit den Vorwahlintentionen konsistent sind. In etwa 75 bis 85 Prozent der Angaben bei der Rückerinnerungsfrage konnte keine Evidenz für ihre Fehlerhaftigkeit gefunden werden. Das bedeutet im Umkehrschluss nicht, dass 75 bis 85 Prozent der Rückerinnerungen korrekt sind. Die Panelmethode ist ein zu krudes und ungenaues Instrument, um die Rückerinnerungen der Befragten verifizieren zu können. Die Panelmethode ist lediglich in der Lage, einen Teil der Rückerinnerungen zu falsifizieren. Doch die vorgefundenen, zum Teil sehr starken Zusammenhänge zwischen den Ergebnissen der Rückerinnerungs- und der Panelmethode deuten darauf hin, dass die Mehrzahl der Befragten in der Lage ist, sich an den Zeitraum ihrer endgültigen Entscheidung korrekt zu erinnern.

Aus diesem Befund allein kann jedoch noch nicht geschlossen werden, dass die Daten der Rückerinnerungsmethode die Veränderungen in der Verteilung der Entscheidungszeitpunkte im Zeitverlauf korrekt widerspiegeln. Das ist der zweite wichtige Befund in diesem Beitrag: Es stimmt, dass Wähler ihre Entscheidungen zunehmend spät treffen, aber die Zunahme später Wahlentscheidungen ist längst nicht so dramatisch, wie es die Daten der Rückerinnerungsmethode nahelegen. Es deutet vieles darauf hin, dass in den Sechziger- und Siebzigerjahren deutlich mehr Wähler ihre Entscheidungen in den letzten Monaten oder letzten Wochen vor der Wahl trafen, als es die Rückerinnerungsmethode suggeriert. Es ist nicht davon auszugehen, dass sich bei den Bundestagswahlen 1965 und 1980 lediglich 5 Pro- 
zent der Wähler in den letzten Wochen vor der Wahl entschlossen. Der tatsächliche Anteil der Spätentscheider lag in diesem Zeitraum um mindestens 20 Prozentpunkte höher. Aus ungeklärten Gründen wies die Rückerinnerungsfrage in früheren Wahlstudien die Tendenz auf, die Mehrzahl der objektiven Spätentscheider als frühe Entscheider zu klassifizieren. In den jüngsten Wahlstudien ist diese systematische Verzerrung in den Antworten auf die Rückerinnerungsfrage nicht mehr zu beobachten. Diese „Normalisierung“ des Antwortverhaltens trug zumindest teilweise zur beobachteten Zunahme später Wahlentscheidungen bei.

Auch die Panelmethode ist nicht frei von Messproblemen, und auch mit ihr wird die Zunahme von nicht entschlossenen Wählern überschätzt, weil der Anteil der unentschlossenen Wähler je nach Erhebungsmodus variiert. Face-to-faceInterviews, früher der Standard-Erhebungsmodus in Wahlstudien, erzeugen einen um etwa 10 Prozentpunkte niedrigeren Anteil unentschlossener Wähler als Telefoninterviews. Das liegt daran, dass die Wahlabsicht in face-to-face-Interviews gestützt abgefragt wird, unter Vorlage eines Listenhefts mit allen Antwortoptionen, in denen aber eine „weiß nicht"-Kategorie in der Regel nicht vorgegeben ist. Diese Methode verleitet dazu, eine Partei zu nennen, auch wenn man zuvor noch keine feste Wahlintention aufwies. In Telefoninterviews wird die Wahlabsicht hingegen durch eine offene Frage erhoben, die eher dazu verleitet, Unentschlossenheit einzugestehen. In Online-Studien, die seit einigen Jahren in der Forschung ebenfalls zunehmende Popularität genießen, wird die Wahlabsicht wieder gestützt abgefragt, jedoch unter expliziter Vorgabe einer „weiß nicht“-Option. Auch aus dieser Methode resultieren hohe Anteile unentschlossener Wähler. Moderne Erhebungsmodi, wie CATI- oder Online-Studien, begünstigen somit die Wahrscheinlichkeit, Befragte als noch nicht entschlossen zu klassifizieren.

Trotz dieser Messprobleme deutet vieles darauf hin, dass der Anteil von bereits „seit langem“ getroffenen Wahlentscheidungen abgenommen hat. Es gestaltet sich jedoch als schwierig, das Ausmaß der Veränderung in Zahlen zu fassen. Wenn man trotz dieser Unwägbarkeiten eine grobe Schätzung abgeben müsste, dann würde die Vermutung des Verfassers lauten, dass der Anteil der „seit langem“ vor der Wahl getroffenen Wahlentscheidungen heutzutage etwa 10 bis 20 Prozentpunkte niedriger ist als noch vor 40 Jahren. Würde man ausschließlich den Daten der Rückerinnerungsmethode vertrauen, läge die Veränderung bei etwa 35 Prozentpunkten. Auch wenn somit die Verschiebung des Zeitpunkts der Wahlentscheidung in Richtung Wahltag nicht so stark ausfällt, wie bislang vermutet, sollten die daraus resultierenden Implikationen für das Wirkungspotenzial von Wahlkämpfen nicht unterschätzt werden. Worauf die moderate Zunahme später Wahlentscheidungen zurückzuführen ist, ist eine andere Frage. Dealignment erscheint noch immer als eine wahrscheinliche Erklärung. Man sollte jedoch auch nicht außer Acht lassen, dass sich die Wahlmöglichkeiten in den vergangenen Jahrzehnten durch die Ausdifferenzierung des Parteiensystems erheblich vergrößerten. Für den Wähler wird es dadurch schwieriger, zwischen ähnlichen Parteien innerhalb eines politischen Lagers eine endgültige Wahlentscheidung zu treffen (hierzu auch Plischke u. Bergmann 2012, S. 510-511).

Die hier vorgestellten Ergebnisse verfügen auch über methodische Implikationen. Sie stellen eine Warnung dar, dass Zeitreihen sozialwissenschaftlicher Indika- 
toren immer ein gewisses Ausmaß an Skepsis entgegengebracht werden sollte, vor allem wenn es sich dabei um subjektive Einschätzungen der Befragten handelt. Häufig ist man als Sozialwissenschaftler darauf angewiesen, auf die zeitliche Vergleichbarkeit von Messwerten zu vertrauen, weil keine alternativen Indikatoren zur Verfügung stehen, die eine Validierung erlauben. In dieser Arbeit war eine solche Validierung ausnahmsweise möglich. Die Ergebnisse machten darauf aufmerksam, dass sich im Zeitverlauf nicht nur die „wahren“ Eigenschaften der Befragten verändern können, sondern auch jene kognitiven oder technischen Prozesse, die für eine Übersetzung der „wahren“ Werte in standardisierte Zahlencodes sorgen.

\section{Literatur}

Bacher, Johann. 2002. Statistisches Matching - Anwendungsmöglichkeiten, Verfahren und ihre praktische Umsetzung in SPSS. ZA-Information 51: 38-66.

Berger, Manfred, Rudolf Wildemann, Uwe Schleth, Max Kaase, Wolfgang G. Gibowski, und Dieter Roth. 1972. Wablstudie 1972. Panel: Voruntersuchung, September-Oktober 1972. ZA-Nr. 0635. Köln: GESIS-Datenarchiv. doi:10.4232/1.10838.

Berger, Manfred, Wolfgang G. Gibowski, Edelgard Gruber, Dieter Roth, Wolfgang Schulte, Max Kaase, Hans-Dieter Klingemann, und Uwe Schleth (Forschungsgruppe Wahlen). 1976. Wahlstudie 1976. Panel: Voruntersuchungen, Mai-Juni 1976, August-September 1976; Nachuntersuchung, Oktober-November 1976. ZA-Nr. 0823. Köln: GESISDatenarchiv. doi:10.4232/1.10839.

Berger, Manfred, Wolfgang G. Gibowski, Dieter Roth, und Wolfgang Schulte. 1983a. Stabilität und Wechsel. Eine Analyse der Bundestagswahl 1980. In Wablen und politisches System. Analysen aus Anlaß der Bundestagswahl 1980, Hrsg. Max Kaase und HansDieter Klingemann, 12-57. Opladen: Westdeutscher Verlag.

Berger, Manfred, Wolfgang G. Gibowski, und Dieter Roth (Forschungsgruppe Wahlen). 1983b. Wahlstudie 1983. Panelstudie. ZA-Nr. 1276. Köln: GESIS-Datenarchiv. doi:10.4232/1.11458.

Berger, Manfred, Wolfgang G. Gibowski, Max Kaase, Hans Dieter Klingemann, Manfred Küchler, Franz U. Pappi, Dieter Roth, und Wolfgang Schulte (Forschungsgruppe Wahlen). 1987. Panelstudie. ZA-Nr. 1537. Köln: GESIS-Datenarchiv. doi:10.4232/1.11459.

Berger, Manfred, Wolfgang G. Gibowski, Dieter Roth, und Wolfgang Schulte (Forschungsgruppe Wahlen). 1987b. Wablstudie 1987. Trenduntersuchungen. ZA-Nr. 1536. Köln: GESIS-Datenarchiv. doi:10.4232/1.1536.

Berger, Manfred, Wolfgang G. Gibowski, Dieter Roth, und Wolfgang Schulte (Forschungsgruppe Wahlen). 1988. Politbarometer 1988. Kumulierter Datensatz. ZA-Nr. 1762. Köln: GESIS-Datenarchiv. doi:10.4232/1.1762.

Berger, Manfred, Matthias Jung, Dieter Roth, Wolfgang G. Gibowski, Max Kaase, HansDieter Klingemann, Manfred Küchler, Franz U. Pappi, und Holli A. Semetko (Forschungsgruppe Wahlen). 1994a. Wahlstudie 1994. Politbarometer West. ZA-Nr. 2546. Köln: GESIS-Datenarchiv. doi:10.4232/1.2546

Berger, Manfred, Matthias Jung, Dieter Roth, Wolfgang G. Gibowski, Max Kaase, HansDieter Klingemann, Manfred Küchler, Franz U. Pappi, und Holli A. Semetko (For- 
schungsgruppe Wahlen). 1994b. Wablstudie 1994. Politbarometer Ost. ZA-Nr. 2559. Köln: GESIS-Datenarchiv. doi:10.4232/1.2559.

Berger, Manfred, Matthias Jung, und Dieter Roth (Forschungsgruppe Wahlen). 1995. Politbarometer Ost 1995. Kumulierter Datensatz. ZA-Nr. 2777. Köln: GESIS-Datenarchiv. doi:10.4232/1.2777.

Chaffee, Steven H., und Rajiv N. Rimal. 1996. Time of Vote Decision and Openness to Persuasion. In Political persuasion and attitude change, Hrsg. Diana C. Mutz, Paul M. Sniderman, und Richard A. Brody, 267-291. Ann Arbor: University of Michigan Press.

Dalton, Russell J. 2012. Apartisans and the changing German electorate. Electoral Studies 31: 35-45.

Dalton, Russell J., und Wilhelm Bürklin. 2003. Wähler als Wandervögel: Dealignment and the German Voter. German Politics \& Society 21: 57-75.

Dalton, Russell J., Ian McAllister, und Martin P. Wattenberg. 2000. The Consequences of Partisan Dealignment. In Parties Without Partisans: Political Change in Advanced Industrial Democracies, Hrsg. Russell J. Dalton und Martin P. Wattenberg, 37-59. Oxford: Oxford University Press.

Deutsches Institut für Volksumfragen (DIVO). 1965. Bundestagswabl 1965. Nachuntersuchung. ZA-Nr. 0314. Köln: GESIS Datenarchiv. doi:10.4232/1.0314.

Erhardt, Klaudia. 1998. Die Unentschlossenen Wähler als Motor zyklischer Wahlabsichtsverläufe. Eine Untersuchung am Beispiel der Rückgewinnung der Wähler durch die CDU. In Politische Meinungsbildung und Wablverhalten: Analysen zum „Superwabljahr“ 1994, Hrsg. Christine Dörner und Klaudia Erhardt, 15-118. Opladen: Westdeutscher Verlag.

Falter, Jürgen, Oscar W. Gabriel, und Hans Rattinger. 1998. Politische Einstellungen, politische Partizipation und Wählerverhalten im vereinigten Deutschland 1998. ZA-Nr. 3066. Köln: GESIS Datenarchiv. doi:10.4232/1.11461.

Falter, Jürgen, Oscar W. Gabriel, und Hans Rattinger. 2002. Politische Einstellungen, politische Partizipation und Wählerverhalten im vereinigten Deutschland. ZA-Nr. 3861. Köln: GESIS Datenarchiv. doi:10.4232/1.11462.

Fazio, Russell H., Jim Blascovich, und Denise M. Driscoll. 1992. On the Functional Value of Attitudes: The Influence of Accessible Attitudes on the Ease and Quality of Decision Making. Personality and Social Psychology Bulletin 18: 388-401.

Forschungsgruppe Wahlen. 1990. Wahlstudie 1990. Politbarometer. ZA-Nr. 1920. Köln: GESIS-Datenarchiv. doi:10.4232/1.1920.

Fournier, Patrick, Richard Nadeau, André Blais, Elisabeth Gidengil, und Neil Nevitte. 2004. Time-of-voting decision and susceptibility to campaign effects. Electoral Studies 23: 661-681.

Fournier, Patrick, Richard Nadeau, André Blais, Elisabeth Gidengil, und Neil Nevitte. 2001. Validation of Time-of-Voting-Decision Recall. Public Opinion Quarterly 65: 95 107.

Fuchs, Dieter, Max Kaase, Hans-Dieter Klingemann, Uwe Schleth und Wolfgang Schulte (Forschungsgruppe Wahlen). 1980. Wahlstudie 1980. Politbarometer. ZA-Nr. 1053. Köln: GESIS-Datenarchiv. doi:10.4232/1.1053.

Himmelweit, Hilde T., Marianne Jaeger Biberian, und Janet Stockdale. 1978. Memory for past Vote: Implications of a Study of Bias in Recall. British Journal of Political Science 8: 365-375. 
Irwin, Galen A., und Joop J. M. Van Holsteyn. 2008. What Are they Waiting for? Strategic Information for Late Deciding Voters. International Journal of Public Opinion Research 20: 483-493.

Juhász, Zoltan. 1993. Wahlabsicht und Rückerinnerung - zwei Angaben zur aktuellen Bewertung der politischen Parteien? In Wahlen in Zeiten des Umbruchs, Hrsg. Oscar W. Gabriel und Klaus G. Troitzsch, 27-50. Frankfurt a. M.: Peter Lang.

Jung, Matthias. 1990. Auf dem Weg zur besseren Datenqualität. Ein Zwischenbericht über die Erfahrungen mit telefonischen Umfragen. In Wablen, Parteieliten, politische Einstellungen: neuere Forschungsergebnisse, Hrsg. Karl Schmitt, 389-412. Frankfurt a. M.: Peter Lang.

Kaase, Max, Hans-Dieter Klingemann, Manfred Küchler, Franz U. Pappi, und Holli A. Semetko (Forschungsgruppe Wahlen). 1990. Wahlstudie 1990. Panelstudie. ZA-Nr.: 1919. Köln: GESIS-Datenarchiv. doi:10.4232/1.11607.

Klingemann, Hans-Dieter, und Franz Urban Pappi. 1969. Bundestagswahl 1969. Voruntersuchung, September 1969 und Nachuntersuchung, Oktober-November 1969. ZA-Nr. 0426. Köln: GESIS Datenarchiv doi:10.4232/1.11456.

Kogen, Lauren, und Jeffrey Gottfried. 2011. I Knew it All Along! Evaluating Time-of-Decision Measures in the 2008 U.S. Presidential Campaign. Political Behavior 34: 719-736.

Krosnick, Jon A. 1999. Survey Research. Annual Review of Psychology 50: 537-567.

Lazarsfeld, Paul F., Bernhard D. Berelson, und Hazel Gaudet. 1944. The People's Choice: How the Voter Makes Up His Mind in a Presidential Campaign. New York: Columbia University Press.

McAllister, Ian. 2002. Calculating or capricious? The new politics of late deciding voters. In Do Political Campaigns Matter? Campaign Effects in Elections and Referendums, Hrsg. David M. Farrell und Rüdiger Schmitt-Beck, 22-39. London: Routledge.

Miller, George A. 1956. The Magical Number Seven, Plus or Minus Two. Some Limits on our Capacity for Processing Information. Psychological Review 63: 81-97.

Noelle-Neumann, Elisabeth. 2001. Die Schweigespirale: Öffentliche Meinung - unsere soziale Haut. München: Langen Müller.

Plasser, Fritz, Peter A. Ulram, und Gilg Seeber. 2003. Erdrutschwahlen: Momentum, Motive und neue Muster im Wahlverhalten. In Wablverhalten in Bewegung. Analysen zur Nationalratswahl 2002, Hrsg. Fritz Plasser und Peter A. Ulram, 97-157. Wien: Schriftenreihe des Zentrums für Angewandte Politikforschung.

Plischke, Thomas, und Michael Bergmann. 2012. Entscheidungsprozesse von Spätentscheidern bei der Bundestagswahl 2009. In Wählen in Deutschland. PVS Sonderheft, Hrsg. Rüdiger Schmitt-Beck, 502-527. Baden-Baden: Nomos.

Plumb, Elizabeth. 1986. Validation of voter recall: Time of electoral decision making. Political Behavior 8: 302-312.

Rattinger, Hans, Sigrid Roßteutscher, Rüdiger Schmitt-Beck, und Bernhard Weßels. 2009a. German Longitudinal Election Study (GLES): Vor- und Nachwahlquerschnitt. ZA-Nr. 5302. Köln: GESIS Datenarchiv. doi:10.4232/1.11373.

Rattinger, Hans, Sigrid Roßteutscher, Rüdiger Schmitt-Beck, und Bernhard Weßels. 2009b. German Longitudinal Election Study (GLES). Rolling-Cross-Section-Wahlkampfstudie mit Nachwahl-Panelwelle. ZA-Nr. 5303. Köln: GESIS-Datenarchiv. doi:10.4232/1.11604. 
Rattinger, Hans, Sigrid Roßteutscher, Rüdiger Schmitt-Beck, und Bernhard Weßels. 2009c. German Longitudinal Election Study (GLES). Wahlkampf-Panel. ZA-Nr. 5305. Köln: GESIS-Datenarchiv. doi:10.4232/1.11131.

Reinemann, Carsten, Marcus Maurer, Thomas Zerback, und Olaf Jandura. 2013. Die Spätentscheider. Medieneinflüsse auf kurzfristige Wablentscheidungen. Wiesbaden: Springer VS.

Schmitt-Beck, Rüdiger. 2003. Kampagnenwandel und Wählerwandel, „Fenster der Gelegenheit" für einflussreichere Wahlkämpfe. In Machtdarstellung und Darstellungsmacht. Beiträge zu Theorie und Praxis moderner Politikvermittlung, Hrsg. Ulrich Sarcinelli und Jens Tenscher, 199-218. Baden-Baden: Nomos.

Schmitt-Beck, Rüdiger, und Thorsten Faas. 2005. Bundestagswahl 2005. Kampagnendynamik - Vor- und Nachwahlstudie. ZA-Nr. 4991. Köln: GESIS-Datenarchiv. doi:10.4232/1.4991.

Schmitt-Beck, Rüdiger, Thorsten Faas, und Christian Holst. 2006. Der Rolling CrossSection Survey - ein Instrument zur Analyse dynamischer Prozesse der Einstellungsentwicklung. Bericht zur ersten deutschen RCS-Studie anlässlich der Bundestagswahl 2005. ZUMA-Nachrichten 58: 13-49.

Schmitt-Beck, Rüdiger und Julia Partheymüller. 2012. Why Voters Decide Late: A Simultaneous Test of Old and New Hypotheses at the 2005 and 2009 German Federal Elections. German Politics 21: 299-316.

Schoen, Harald. 2003. Wählerwandel und Wechselwahl: Eine vergleichende Untersuchung. Wiesbaden: Westdeutscher Verlag.

Sniderman, Paul M., Richard A. Brody, und Philip E. Tetlock. 1991. Reasoning and Choice: Explorations in Political Psychology. New York: Cambridge University Press.

van der Eijk, Cornelis, und Broer Niemoller. 1983. Electoral change in the Netherlands: empirical results and methods of measurement. Amsterdam: CT-Press.

Waldahl, Ragnar, und Bernt Aardal. 2000. The Accuracy of Recalled Previous Voting: Evidence from Norwegian Election Study Panels. Scandinavian Political Studies 23: 373389.

Weßels, Bernhard. 2005. Nachwahlstudie zur Bundestagswabl 2005. Testmodul zur 3. Welle der ,Comparative Study of Electoral Systems' (CSES). ZA-Nr. 4559. Köln: GESIS-Datenarchiv. doi:10.4232/1.4559.

\section{Autorenangaben}

Dr. Thomas Plischke

Universität Mannheim, Lehrstuhl für Vergleichende Politische Verhaltensforschung, A 5,6 , 68131 Mannheim, thomas.plischke@uni-mannheim.de 